J. Lake Sci.(湖泊科学), 2020, 32(2): 309-324

DOI 10. 18307/2020. 0203

(c) 2020 by Journal of Lake Sciences

\title{
持久性有机污染物在中国湖库水体中的污染现状及分布特征
}

\author{
陶玉强 ${ }^{1,2 * *}$, 赵䢒涵 ${ }^{3}$ \\ (1: 河海大学海洋学院, 南京 210098) \\ (2: 中国科学院南京地理与湖泊研究所湖泊与环境国家重点实验室,南京 210008) \\ (3: 南京农业大学资源与环境科学学院,南京 210095)
}

\begin{abstract}
摘 要: 持久性有机污染物 $(\mathrm{POPs})$ 在环境中广泛且持久存在,多数具有致畸、致癌、致突变等危害. 中国是全球湖泊密度 较大的国家之一, 湖泊在供水、渔业、维护生态系统多样性等众多方面发挥了重要作用. 湖泊是 POPs 的重要归宿之一. 湖 泊水体中的 POPs 可被水生生物吸收利用并传递, 对生态系统及人体健康构成较大危害. 因此湖泊水体中 POPs 的分布水 平对周围的生态系统及人体健康有非常重要的影响. 本文收集、分析了 2003 年至 2019 年 2 月公开发表的 80 篇文献中的 中国湖泊水体 $\mathrm{POPs}$ 数据, 包括多环芳烃 $(\mathrm{PAHs})$ 、六六六类农药 $(\mathrm{HCHs}) 、$ 滴滴涕类农药 $(\mathrm{DDX}) 、$ 多氯联苯 $(\mathrm{PCBs}) 、$ 多溴 联苯醚 (PBDEs)、多氯联苯梄类( PCDEs)、全氟化合物 (PFCs) 及邻苯二甲酸酯类 (PAEs), 发现: 目前中国已有水体 POPs 研究数据的湖泊共有 49 个, 涉及 19 个省、直辖市、自治区, 研究区域主要在东部地区 $(n=32)$, 云贵高原 $(n=14)$ 和青藏一 新疆湖区 $(n=3)$ 少有研究, 其它地区鲜有研究; PAHs $、 \mathrm{HCHs}$ 和 DDX 是研究最多的三类 POPs, 在中国湖泊水体中平均浓 度分别为 $360.0 \pm 433.8 \mathrm{ng} / \mathrm{L}(n=26) 、 12.8 \pm 23.5 \mathrm{ng} / \mathrm{L}(n=30) 、 12.8 \pm 24.6 \mathrm{ng} / \mathrm{L}(n=24)$; 不同湖区间、不同种类 POPs 以及 不同时间相同 POPs 检出值差距均较大, 东部湖区水体 POPs 浓度高于其它湖区; 从时间角度看, 虽然这三类 POPs 各自占 总污染物量比例在 2010 年前后无较大变化, 但 2010 年后的浓度与 2010 年以前相比有所下降, 说明中国履行《关于持久 性有机污染物 ( POPs) 的斯德哥尔摩公约》内容以及对 PAHs 的管控减排已显成效; POPs 在中国湖泊水体中的浓度与季 节、水体深度 (上覆水与间隙水)、采样点位置 ( 入湖口、湖心及出水口) 以及人为活动 (农业耕作、生活排污、工业污水及 码头船只往来等) 存在一定关系, 但并非完全受这些因素影响, 还与 POPs 种类、所处区域的地理位置等诸多因素有关. 本 文基于大量已发表文献中的研究数据, 揭示了中国湖泊水体 POPs 的污染现状及分布特征, 可为中国 POPs 的污染控制及 湖泊保护提供一定的科学依据.
\end{abstract}

关键词: 持久性有机污染物; 中国湖泊;湖泊水体;污染现状;分布特征

\section{Occurrence and distribution of persistent organic pollutants in water of Chinese lakes and reservoirs *}

\author{
TAO Yuqiang ${ }^{1,2 * *} \&$ ZHAO Ruihan ${ }^{3}$ \\ (1: College of Oceanography, Hohai University, Nanjing 210098, P.R. China) \\ (2: State Key Laboratory of Lake Science and Environment, Nanjing Institute of Geography and Limnology, Chinese Academy \\ of Sciences, Nanjing 210008, P.R.China) \\ (3: College of Resources and Environmental Sciences, Nanjing Agricultural University, Nanjing 210095, P.R.China)
}

Abstract : Persistent organic pollutants (POPs) are widespread and persistent in the environment, and most of them are teratogenic, carcinogenic, and mutagenic. China is one of the countries with a high density of lakes in the world. Lakes play an important role in many aspects such as water supply, fisheries, and maintenance of ecosystem diversity. In addition, lakes are important sinks of POPs. The POPs in lake water can absorb and be utilized by aquatic organisms, which poses great risk to ecosystems and human

* 2019-06-18 收稿; 2019-08-29 收修改稿.

江苏省杰出青年基金项目 (BK20170049)、国家自然科学基金项目 (41977330)、中国科学院青年创新促进会优秀会 员专项、国家科技基础性工作专项 (2015FY110900-03) 和中国科学院南京地理与湖泊研究所交叉团队项目 ( NIGLAS2016TD01) 联合资助.

** 通信作者; E-mail: yqtao@ hhu.edu.cn. 
health. Therefore, the distribution of POPs in lake water has an important impact on the surrounding ecosystem and human health. We collected 80 articles on the topic of POPs in Chinese lake water published from 2003 to February 2019. We collected and analyzed the published data of polycyclic aromatic hydrocarbons (PAHs), hexachlorocyclohexanes ( HCHs), dichlorodiphenyltrichloroethane (DDX), polychlorinated biphenyls (PCBs), polybrominated diphenyl ethers (PBDEs), polychlorinated diphenyl ethers (PCDEs), perfluorinated compounds (PFCs), and phthalate esters (PAEs). The POPs in Chinese lake water have been investigated in 49 lakes, involving 19 provinces, municipalities and autonomous regions in China, mainly located in the eastern region $(n=32)$, and Yunnan-Guizhou Plateau $(n=14)$. There were fewer studies in the Qinghai-Xinjiang Lake District $(n=3)$, and other areas. Among them, PAHs, HCHs and DDX were the most studied POPs. Their average concentrations of $\Sigma$ PAHs, $\Sigma H C H s$, and $\Sigma$ DDX in Chinese lake water were $360.0 \pm 433.8 \mathrm{ng} / \mathrm{L}(n=26), 12.8 \pm 23.5 \mathrm{ng} / \mathrm{L}(n=30)$, and $12.8 \pm 24.6 \mathrm{ng} / \mathrm{L}(n=24)$. The concentrations of POPs in water of eastern lakes were higher than those in lakes in other areas. Although the relative proportions of $\Sigma$ PAHs, $\Sigma$ HCHs and $\Sigma$ DDX to the concentration of total pollutants did not change significantly before and after the year of 2010, their concentrations decreased, suggesting that the compliance with the Stockholm Convention on persistent organic pollutants (POPs) and the mitigation of PAHs were effective in China. The depth of water (overlying water and interstitial water), sampling location ( the inlet and outlet of lakes and within the lakes) were important factors for concentrations of POPs in Chinese lake water. The concentrations of POPs in Chinese lake water were correlated with human activities such as agricultural farming, domestic sewage, industrial sewage, and docks, and the type of POPs and geographical location of the studied area. Based on the research data collected from a large number of published literatures, this review revealed the distribution characteristics of POPs in Chinese lake water, and provided a scientific basis for the control and mitigation of POPs in Chinese lakes.

Keywords: Persistent organic pollutants; Chinese lake; lake water; occurrence; distribution charactersitics

持久性有机污染物 (persistent organic pollutants, 简称 POPs) 主要指能够长距离迁移并长期存在于环境 介质 (大气、水、土壤、生物体等) 中, 且对人类健康和环境具有严重危害的天然或人工合成的有机污染物 质 ${ }^{[1]}$. 因其具有持久性、高毒性、难降解、生物累积性、可通过各种环境介质广泛传播等特性, POPs 已成为全 球关注的热点. 2001 年 5 月 22-23 日,91 个国家政府和欧洲共同体在瑞典首都斯德哥尔摩共同签署了《关 于持久性有机污染物 (POPs) 的斯德哥尔摩公约》, 作为控制 POPs 的生产、进出口、使用和处理以及保护人 类健康和环境免受其危害的全球行动条约. POPs 来源广泛, 在环境各介质中普遍存在,进人环境后, 可通过 直接排放、干湿沉降及地表径流等方式进人水环境 ${ }^{[2]}$. 湖泊是自然界水环境不可或缺的一员, 是陆地表层系 统各要素相互作用的节点, 是地球上重要的淡水资源库、洪水调蓄库和物种基因库, 与人类生产与生活息息 相关, 在维系流域生态平衡、满足生产生活用水、减轻洪涝灾害和提供丰富水产等方面发挥着不可替代的 作用 ${ }^{[3]}$.

中国是一个湖泊众多的国家. 湖泊不仅具有供水、调蓄洪水、调节气候、渔业、旅游等功能, 还在维持区 域生态系统平衡和保育生物多样性等方面发挥着重要作用, 是支撑中国经济和社会发展的重要资源之一 ${ }^{[4]}$. 然而随着中国经济、社会、人类生产生活方式等的快速发展,众多淡水湖泊包括其水源地受到了不同程度的 POPs 污染, 严重影响了饮用水及湖泊生态系统的安全, 威胁着人体健康. 与其它陆地水体相比, 湖泊水流迟 缓且换水周期长, 对污染物的稀释能力比较弱 ${ }^{[3]}$, 水体环境相对稳定. 因此, 湖泊水体能较好地反映流域乃 至区域内 POPs 污染信息,包括 POPs 的污染水平、不同区域及不同时间的 POPs 排放情况以及分布特征等. 因此,加强对湖泊水体中 POPs 污染现状及分布规律等的研究具有重要意义.

虽然已有较多学者陆续研究了 POPs 在中国湖泊、水库水体中的污染水平及分布特征, 但以往的研究多 局限于单个或少数几个湖泊, 且研究时间尺度较短、时间不连续, 缺少对中国湖泊水体 POPs 污染现状及分 布特征的全面认识,一定程度上阻碍了对中国湖泊水体 POPs 污染风险的认识及其科学管理. 本文收集了截 止到 2019 年 2 月公开发表的 80 篇关于中国湖泊水体多环芳烃( PAHs)、有机氯农药 (OCPs)、多氯联苯 (PCBs)、多溴联苯梄( PBDEs)、全氟磺基化合物 (PFOSs) 等 POPs 的论文及其数据, 分析了这些 POPs 在中 国湖泊水体中的污染现状及分布特征. 此研究可为合理评估中国湖泊水体中 POPs 污染风险提供一些支撑 数据, 并可为中国湖泊水体 POPs 的科学管理提供一些参考依据.

\section{1 材料与方法}

通过中国知网、万方数据库、维普科技期刊数据库、Web of Science 等学术搜索引擎收集了截止 2019 年 
2 月公开发表的关于中国湖泊水体中 16 种优先控制的多环芳烃、有机氯农药 (其中 HCHs 包括 $\alpha-\mathrm{HCH} 、 \beta-$ $\mathrm{HCH} 、 \gamma-\mathrm{HCH}$ 和 $\delta-\mathrm{HCH}$, 滴滴涕类农药 (DDX) 主要收集 $p, p^{\prime}$-和 $o, p^{\prime}$-DDE、DDD 与 DDT)、多氯联苯、多溴联 苯醚、全氟烷基化合物等 POPs 分布状况的 80 余篇论文. 搜集的论文涉及到 49 个中国湖泊,分布在中国的 17 个省市区, 涉及到的各个湖泊基本信息及所处的经纬度如附录 1 所示. 统计了各类 POPs 在湖泊水体分 布的时间、空间状况及分布趋势,并将所收集数据按湖泊和污染物种类取均值,用以比较观察其中的差异. 其中 $n$ 在整体比较中代表已有研究所涉及的湖泊个数, 在各个湖泊分析时代表所采集样品个数, 误差是基 于以参数形式给出的整个样本总体计算标准偏差, 用 STDEVP 函数求得; 原文献中未说明原始数据, 无法计 算标准偏差处取平均数值的 $5 \%$ 代替; 为便于与仅采集上覆水的研究结果相比较, 本文中各湖泊的污染物均 值计算与数值比较均只取上覆水检测结果数值.

\section{2 中国湖泊水体中 POPs 的污染现状}

\section{1 中国湖泊水体中 PAHs 的污染现状}

从空间分布上看,截止 2019 年 2 月关于中国湖泊水体中 PAHs 的研究主要集中在东部地区 ( 22 个湖 泊), 青藏一新疆湖区与云贵高原湖区少有研究 (4 个湖泊). 已报道的 26 个中国湖泊水体中 16 种优先控制 的 PAHs 总浓度 $\left(\sum \mathrm{PAH}_{16}\right)$ 范围为 4 9847 $\mathrm{ng} / \mathrm{L}$, 平均浓度为 $360.0 \pm 433.8 \mathrm{ng} / \mathrm{L}(n=26)$ (图 1). 各湖泊水体 中 $\Sigma \mathrm{PAH}_{16}$ 高低顺序依次为西湖 $(2173.8 \pm 1475.8 \mathrm{ng} / \mathrm{L})(n=2)>$ 南四湖 $(974.5 \pm 1043.2 \mathrm{ng} / \mathrm{L})(n=1)>$ 太湖 $(752.4 \pm 987.3 \mathrm{ng} / \mathrm{L})(n=10)>$ 玄武湖 $(640.0 \pm 62.7 \mathrm{ng} / \mathrm{L})(n=1)>$ 百花湖 $(600.7 \pm 129.4 \mathrm{ng} / \mathrm{L})(n=1)$, 麓湖 水体内 $\Sigma \mathrm{PAH}_{16}$ 最低, 平均浓度为 $49.8 \pm 17.4 \mathrm{ng} / \mathrm{L}(n=1)$. 东部地区湖泊水体 $\Sigma \mathrm{PAH}_{16}$ 平均浓度为 $379.2 \pm$ $461.0 \mathrm{ng} / \mathrm{L}(n=22)$, 云贵高原湖泊水体 $\Sigma \mathrm{PAH}_{16}$ 为 $303.6 \pm 216.6 \mathrm{ng} / \mathrm{L}(n=3)$, 青藏一新疆湖泊水体 $\Sigma \mathrm{PAH}_{16}$ 平 均浓度为 $189.7 \pm 9.5 \mathrm{ng} / \mathrm{L}(n=1)$. 东部地区湖泊水体 $\Sigma \mathrm{PAH}_{16}$ 高于云贵高原湖泊水体 $\Sigma \mathrm{PAH}_{16}$, 远高于青藏一 新疆湖泊水体 $\Sigma \mathrm{PAH}_{16}$.

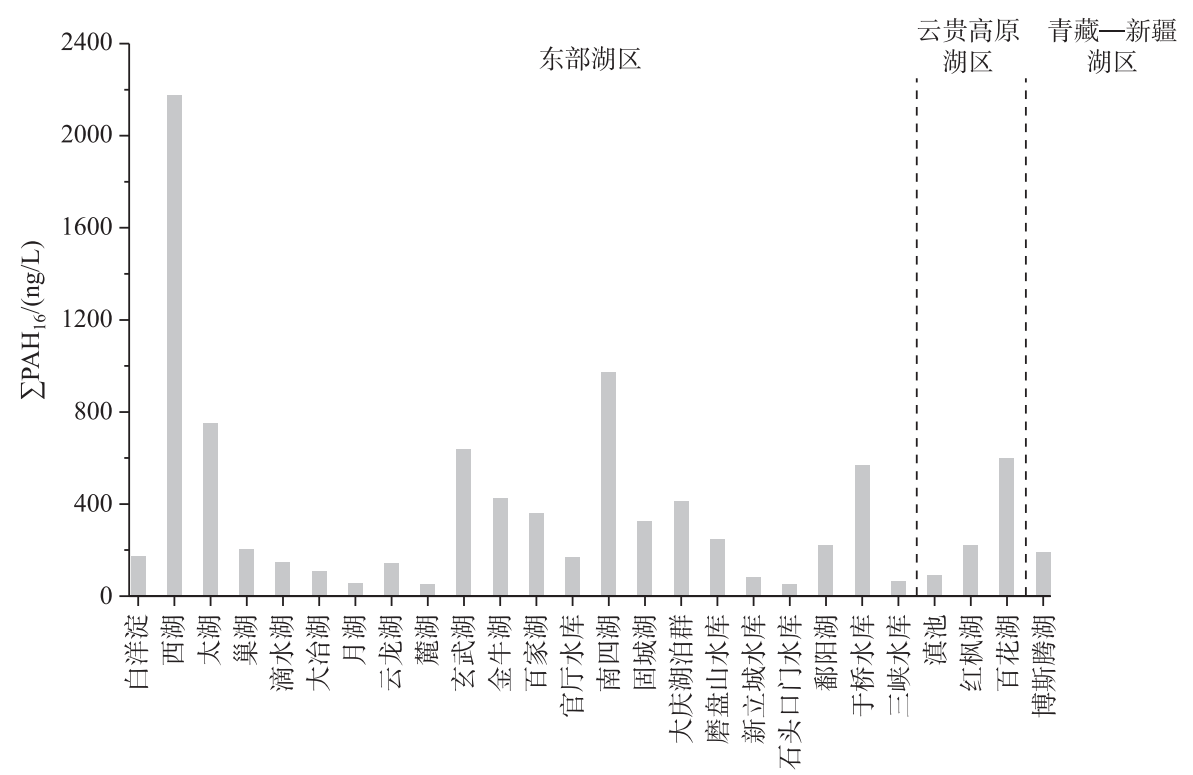

图 1 中国各湖泊、水库水体中 $\Sigma \mathrm{PAH}_{16}$ 平均浓度 ${ }^{[5-11,15,16,22,24,25,27,30,34,49-63,79-83,86-88,95-98]}$

Fig. 1 The average concentration of $\Sigma \mathrm{PAH}_{16}$ in water of Chinese lakes and reservoirs

高秋生等 ${ }^{[5]} 2016$ 年 3 月在白洋淀采集了 15 个样点的水体样品, 并对其中的 16 种优先控制的 PAHs 浓 度进行了测定, 发现 $\Sigma \mathrm{PAH}_{16}$ 的浓度范围为 $71.3 \sim 228.3 \mathrm{ng} / \mathrm{L}$, 平均浓度为 $133.4 \pm 47.3 \mathrm{ng} / \mathrm{L}$, 与其它湖区相 比, 白洋淀 $\Sigma \mathrm{PAH}_{16}$ 浓度处于较低污染水平. 从各个采样点分布位置来看, 光淀张庄 $(229.6 \mathrm{ng} / \mathrm{L})$ 湖区 
$\Sigma \mathrm{PAH}_{16}$ 浓度最高, 其次为安新大桥 $(218.4 \mathrm{ng} / \mathrm{L})$, 郭里口 $(73.2 \mathrm{ng} / \mathrm{L})$ 湖区 $\Sigma \mathrm{PAH}_{16}$ 浓度最低. 根据多环芳烃 中苯环的个数将其进行分类, 3 环和 4 环浓度最高, 分别占 $\Sigma \mathrm{PAH}_{16}$ 总浓度的 $41.2 \%$ 和 $21.4 \%$, 其次是五环 (18.6\%), 检测浓度最低的为六环, 占总浓度的 7.5\%, 总体来看, 高环芳烃 (5 6 环) 单体平均浓度普遍低于中 低环芳烃 (2 4 环). 就 PAH 单体浓度水平而言, 浓度较高的 PAH 单体为萘 ( Nap), 其平均浓度为 $15.1 \mathrm{ng} / \mathrm{L}$ 、 其次为菲 (Phe) (12.4 ng/L) 和范 (Acy) (12.2 ng/L), 较低浓度的单体为苯并 $(\mathrm{a})$ 蒽 $(\mathrm{BaA})(2.2 \mathrm{ng} / \mathrm{L})^{[5]}$, 与 历史数据相比较,此次 $\Sigma \mathrm{PAH}_{16}$ 浓度低于 2009 年秋季的 $454.1 \pm 200.3 \mathrm{ng} / \mathrm{L}^{[6]}$, 高于 2015 年 8 月的 $76.2 \pm 88.2$ $\mathrm{ng} / \mathrm{L}^{[7]}$. 张家泉等 2015 年 8 月采集大冶湖水样 8 个, 研究发现水体中 $\Sigma \mathrm{PAH}_{16}$ 浓度范围为 $27.9 \sim 243.0 \mathrm{ng} / \mathrm{L}$, 平均浓度为 $107.8 \mathrm{ng} / \mathrm{L}$, 就 $\mathrm{PAH}$ 单体的浓度水平而言, 主要以 2 环、4 环以及 5 环 $\mathrm{PAH}_{16}$ 为主, 占水体中 $\Sigma \mathrm{PAH}_{16}$ 总浓度的 68\% 97.6\%,3 环以及 6 环 PAHs 浓度极低, 基本可以忽略不计, 荟( Nap)、苯并( b) 苂蒽 $(\mathrm{BbF})$ 和苯并 $(\mathrm{b})$ 苂葋 $(\mathrm{BaP})$ 是水体中检出浓度最高的 3 种单体, $\mathrm{Nap}(22.7 \mathrm{ng} / \mathrm{L})>\mathrm{BbF}(19.9 \mathrm{ng} / \mathrm{L})>\mathrm{BaP}$ $(18.7 \mathrm{ng} / \mathrm{L})$, Acy 在大冶湖水体中检出最少, 平均浓度仅为 $0.47 \mathrm{ng} / \mathrm{L}^{[8]}$, 与其它湖泊相比, 大治湖水体 $\Sigma \mathrm{PAH}_{16}$ 浓度处于较低污染水平. Qin 等 2009 年 8 月在巢湖 15 个采样点进行现场采样, 检测发现 $\Sigma P A H_{16}$ 总 平均浓度为 $170.7 \pm 70.8 \mathrm{ng} / \mathrm{L}$, 范围为 $95.6 \sim 370.1 \mathrm{ng} / \mathrm{L}$, 其中, 4 个人湖河流样点 $\Sigma \mathrm{PAH}_{16}$ 的平均浓度为 $267.3 \pm 80.0 \mathrm{ng} / \mathrm{L}, 11$ 个湖泊样点的 $\Sigma \mathrm{PAH}_{16}$ 平均浓度为 $150.3 \pm 32.9 \mathrm{ng} / \mathrm{L}$, 人湖河流处 $\Sigma \mathrm{PAH}_{16}$ 浓度高于湖泊 水体. PAHs 在巢湖水体中的浓度以低环 $(157.4 \pm 57.3 \mathrm{ng} / \mathrm{L})$ 为主, 其次是中环 $(11.3 \pm 16.0 \mathrm{ng} / \mathrm{L})$, 浓度最低 的是高环 $(0.83 \pm 0.38 \mathrm{ng} / \mathrm{L})$. 各个 PAH 单体以 Nap 浓度最高, 其浓度范围为 $34.2 \sim 105.6 \mathrm{ng} / \mathrm{L}$, 平均浓度为 $68.8 \pm 24.0 \mathrm{ng} / \mathrm{L}$, 其次是 $\mathrm{Phe}$, 其浓度范围为 $28.7 \sim 100.5 \mathrm{ng} / \mathrm{L}$, 平均浓度为 $42.7 \pm 19.2 \mathrm{ng} / \mathrm{L}$, 浓度最低的是 Bap, 其浓度范围为 $0.00 \sim 0.36 \mathrm{ng} / \mathrm{L}$, 平均浓度为 $0.09 \pm 0.10 \mathrm{ng} / \mathrm{L}^{[9]}$, 此研究中 $\Sigma \mathrm{PAH}_{16}$ 浓度略低于 Qin 等 2010 年 5 月一 2011 年 4 月检测结果 $(173.5 \pm 132.9 \mathrm{ng} / \mathrm{L})^{[10]}$, 低于沈登辉 2012 年 12 月- 2013 年 8 月平均浓度 $(495.5 \pm 24.8 \mathrm{ng} / \mathrm{L})^{[11]}$. 总体来看, 中国湖泊水体中 $\mathrm{PAHs}$ 主要以中低环 ( $2 \sim 4$ 环) 为主, 高环 ( $5 \sim 6$ 环) 浓度 占总浓度比例较低, 其中 Nap 检出浓度最高, 其它 PAH 单体的浓度随时间、湖泊所处区域的不同而不同; 从 时间变化趋势来看, 中国湖泊水体中 $\Sigma \mathrm{PAH}_{16}$ 浓度在 2010 年及以前平均浓度为 $537.7 \pm 729.9 \mathrm{ng} / \mathrm{L}(n=13)$, 2010 年后则为 $322.5 \pm 340.1 \mathrm{ng} / \mathrm{L}(n=20)$, 近年来总体呈下降趋势.

\section{2 中国湖泊水体中 OCPs 的污染现状}

OCPs 主要分为以环戊二烯为原料和以苯为原料的两大类 ${ }^{[12]}$, 其中 HCHs 和 DDX 作为有机氯农药的主 要品种, 被重点监测. 各湖泊水体 $\mathrm{HCH}_{4}$ (包括 $\alpha-\mathrm{HCH} 、 \beta-\mathrm{HCH} 、 \gamma-\mathrm{HCH} 、 \delta-\mathrm{HCH}$ ) 和 DDX (包括 $p, p^{\prime}$-和 $o, p^{\prime}-$ DDE、DDD 与 DDT) 的分布状况如图 3 所示. 中国湖泊水体 $\Sigma \mathrm{HCH}_{4}$ 浓度范围为 $0.25 \sim 195 \mathrm{ng} / \mathrm{L}$, 平均浓度为 $12.8 \pm 23.5 \mathrm{ng} / \mathrm{L}(n=30)$ ( 图 $2 \mathrm{a}$ ), 东部地区湖泊水体中 $\Sigma \mathrm{HCH}_{4}$ 浓度为 $19.6 \pm 32.6 \mathrm{ng} / \mathrm{L}(n=14)$, 云贵高原湖区 湖泊水体中 $\Sigma \mathrm{HCH}_{4}$ 浓度为 $6.6 \pm 5.2 \mathrm{ng} / \mathrm{L}(n=13)$, 青藏一新疆湖区湖泊水体中 $\Sigma \mathrm{HCH}_{4}$ 浓度为 $7.9 \pm 7.3 \mathrm{ng} / \mathrm{L}$ $(n=3)$, 东部湖区湖泊水体 $\Sigma \mathrm{HCH}_{4}$ 浓度略高于云贵高原与青藏一新疆湖区水体 $\Sigma \mathrm{HCH}_{4}$ 浓度, 单个湖泊水体 $\Sigma \mathrm{HCH}_{4}$ 浓度高低顺序依次是太湖 $(119 \pm 118 \mathrm{ng} / \mathrm{L})(n=2)>$ 微山湖 $(107.0 \pm 5.4 \mathrm{ng} / \mathrm{L})(n=1)>$ 贡湖 $(23.4 \pm 1.2 \mathrm{ng} /$ $\mathrm{L})(n=1)>$ 博斯腾湖 $(18.1 \pm 0.9 \mathrm{ng} / \mathrm{L})(n=1)>$ 西湖 $(17.3 \pm 2.0 \mathrm{ng} / \mathrm{L})(n=2)>$ 其它湖泊. 水体 $\Sigma$ DDX 浓度范 围为 $0.047 \sim 217.6 \mathrm{ng} / \mathrm{L}$, 平均浓度为 $12.8 \pm 24.6 \mathrm{ng} / \mathrm{L}(n=24)$ (图 $2 \mathrm{~b}$ ). 东部地区湖泊水体 $\Sigma D D X$ 浓度为 $21.2 \pm 30.8 \mathrm{ng} / \mathrm{L}(n=13)$, 云贵高原湖区水体 $\Sigma \mathrm{DDX}$ 浓度为 $3.4 \pm 5.6 \mathrm{ng} / \mathrm{L}(n=8)$, 青藏一新疆湖区水体 $\Sigma \mathrm{DDX}$ 浓度为 $1.8 \pm 2.1 \mathrm{ng} / \mathrm{L}(n=3)$, 东部地区湖泊水体 $\Sigma \mathrm{DDX}$ 浓度高于其它两个地区湖泊水体中的浓度. 中国湖 泊水体中 $\Sigma$ DDX 浓度高低顺序依次是太湖 $(97 \pm 4.6 \mathrm{ng} / \mathrm{L})(n=2)>$ 微山湖 $(96 \pm 4.8 \mathrm{ng} / \mathrm{L})(n=1)>$ 西湖 $(29.5 \pm 11.8 \mathrm{ng} / \mathrm{L})(n=2)>$ 红枫湖 $(17.5 \pm 0.88 \mathrm{ng} / \mathrm{L})(n=1)>$ 汀汊湖 $(15.4 \pm 3.5 \mathrm{ng} / \mathrm{L})(n=2)$, 其余湖泊水体 $\sum \mathrm{DDX}$ 平均浓度较低 $(11.7 \sim 0.27 \mathrm{ng} / \mathrm{L})$.

Dai 等 ${ }^{[13]} 2008$ 年 7 月在白洋淀设置 15 个采样点, 采集水样并对其中的 OCPs 进行定性与定量分析, 检 测发现: 白洋淀水体中 $\Sigma$ OCPs 浓度范围为 $11.4 \sim 30.4 \mathrm{ng} / \mathrm{L}$, 平均浓度为 $17.5 \pm 4.8 \mathrm{ng} / \mathrm{L}$, 在 OCPs 中, $\Sigma H C H s$ 浓度范围为 $3.1 \sim 10.6 \mathrm{ng} / \mathrm{L}$ (平均浓度为 $6.2 \pm 2.3 \mathrm{ng} / \mathrm{L}$ ), $\Sigma \mathrm{DDX}$ 浓度范围为 $4.1 \sim 20.6 \mathrm{ng} / \mathrm{L}$ (平均浓度为 $11.3 \pm$ $4.0 \mathrm{ng} / \mathrm{L})$. 其中, $\mathrm{HCH}$ 各异构体中以 $\beta-\mathrm{HCH}$ 浓度最高, 浓度范围为 $1.3 \sim 6.0 \mathrm{ng} / \mathrm{L}$, 平均浓度为 $3.7 \pm 1.4 \mathrm{ng} / \mathrm{L}$, 占 水体 $\Sigma \mathrm{HCH}_{4}$ 浓度的 $60 \%$, 其次是 $\alpha-\mathrm{HCH}$ (平均浓度为 $1.1 \pm 0.63 \mathrm{ng} / \mathrm{L}$ ), 浓度最低的是 $\delta-\mathrm{HCH}$ (平均浓度为 $0.41 \pm 0.15 \mathrm{ng} / \mathrm{L})$. 各 DDX 的浓度高低顺序为: DDE $\left(o, p^{\prime}-\mathrm{DDE}\right.$ 和 $p, p^{\prime}-\mathrm{DDE}$ 的总和, 平均浓度为 $\left.4.6 \mathrm{ng} / \mathrm{L}\right)>$ 

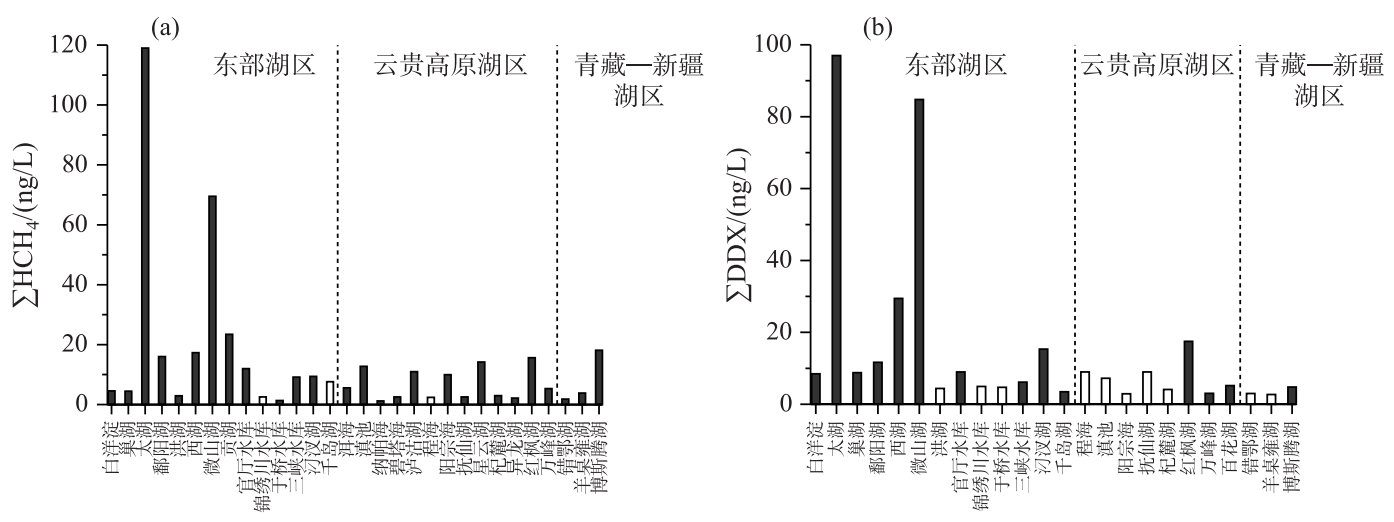

图 2 中国各湖泊、水库水体中 $\Sigma \mathrm{HCH}_{4}(\alpha-\mathrm{HCH} 、 \beta-\mathrm{HCH} 、 \gamma-\mathrm{HCH} 、 \delta-\mathrm{HCH}$ 之和 $)$

平均浓度 $(\mathrm{a})^{[13-18,26,32,62,66-70,72-78,84-85,89-93,94,96,99]}$ 和 $\Sigma$ DDX $\left(p, p^{\prime}-\right.$ 和 $o, p^{\prime}-\mathrm{DDE}$ 、DDD 和 DDT 之和)

平均浓度 $(\mathrm{b})^{[13,15-18,26-27,32,62,67-75,77,78,85,89-94,96,99]}$

（黑色柱代表实际浓度;白色柱代表实际浓度的 10 倍; 图 a 中的鄱阳湖 $2 \mathrm{HCH}_{4}$ 平均

浓度为统计时因缺少原始数据, 故取文献 [16]浓度范围上下限数值的中值;

图 b 中于桥水库和程海的 $\Sigma D D X$ 为实际浓度的 100 倍)

Fig.2 The average concentrations of $\Sigma \mathrm{HCH}_{4}(\mathrm{a})$ and $\Sigma$ DDX (b) in water of Chinese lakes and reservoirs

$\operatorname{DDD}\left(o, p^{\prime}\right.$-DDD 和 $p, p^{\prime}$-DDD 的总和, 平均浓度为 $\left.4.6 \mathrm{ng} / \mathrm{L}\right)>\mathrm{DDT}\left(o, p^{\prime}\right.$-DDT 和 $p, p^{\prime}$-DDT 的总和, 平均浓度 为 $2.60 \mathrm{ng} / \mathrm{L}), \mathrm{DDE}$ 和 DDD 分别占水中 $\sum \mathrm{DDX}$ 的 $41 \%$ 和 $40 \%{ }^{[13]}$, 就 HCHs 与 DDX 浓度而言, 此研究结果 中 $\mathrm{HCHs}$ 浓度略低于 $\mathrm{Li}$ 等 2003 年 7 月的 $0.77 \sim 14.2 \mathrm{ng} / \mathrm{L}^{[14]}$, 高于 $\mathrm{Hu}$ 等 2007 年 7 月的 $2.1 \pm 0.8 \mathrm{ng} / \mathrm{L}^{[15]}$; $\sum D D X$ 浓度高于 2007 年 7 月的 $2.4 \pm 0.6 \mathrm{ng} / \mathrm{L}^{[15]}$, 总体来看, 白洋淀 DDX 与 $\mathrm{HCHs}$ 浓度水平近年来略有下 降,与其它研究相比,白洋淀水体 HCHs 污染处于中等水平, DDX 处于较高污染水平. Zhi 等 2012 年 7 月在 鄱阳湖丰水期共采集 40 个水样 (采样点主要集中在鄱阳湖北部), 对其 OCPs 进行检测发现: $20 \mathrm{CPs}$ 总浓度 范围为 $19.1 \sim 111.8 \mathrm{ng} / \mathrm{L}$, 其中 $\Sigma \mathrm{HCH}_{4}$ 浓度为 $4.4 \sim 59.7 \mathrm{ng} / \mathrm{L}$, 占 $\Sigma O C P s$ 总浓度的 $24.7 \%, \Sigma D D X$ 浓度为 $2.3 \sim$ $33.4 \mathrm{ng} / \mathrm{L} ; \beta-\mathrm{HCH}$ 和 $p, p^{\prime}$-DDT 是主要污染物, $\beta-\mathrm{HCH}$ 的浓度范围为 $1.1 \sim 51.4 \mathrm{ng} / \mathrm{L}, p, p^{\prime}$-DDT 的浓度范围为 $2.0 \sim 33.0 \mathrm{ng} / \mathrm{L}^{[16]} ; \Sigma \mathrm{HCH}_{4}$ 与 $\Sigma \mathrm{DDX}$ 浓度均高于龙智勇 2009 年 3 月检测结果 $(\mathrm{HCHs}$ 浓度范围为 $\mathrm{ND} \sim 0.03$ $\mathrm{ng} / \mathrm{L}$, 平均为 $0.006 \pm 0.011 \mathrm{ng} / \mathrm{L}$; DDX 浓度范围为 $3.6 \sim 52.0 \mathrm{ng} / \mathrm{L}$, 平均为 $\left.11.7 \pm 12.0 \mathrm{ng} / \mathrm{L}^{[17]}\right)$. Yang 等 2003 年 8-10 月在中国云南 11 个高原湖泊共设置 14 个采样点 (除滇池设置 4 个采样点外, 其余湖泊均在湖中心 设置 1 个), 并对水样中的 OCPs 包括 DDX、HCHs、HCB 等浓度进行测定, 发现: 11 个被检测湖泊中, 星云湖 水体 $\Sigma O C P s$ 浓度最高 $(14.2 \mathrm{ng} / \mathrm{L}$ ), 其次是滇池 (浓度范围为 $6.7 \sim 24.5 \mathrm{ng} / \mathrm{L}$, 平均 $13.7 \mathrm{ng} / \mathrm{L}$ ) 和沾沽湖 $(11.0$ $\mathrm{ng} / \mathrm{L})$, 程海最低 $(0.38 \mathrm{ng} / \mathrm{L}), p, p^{\prime}-\mathrm{DDE}$ 的浓度范围为 $\mathrm{ND} \sim 1.9 \mathrm{ng} / \mathrm{L}$ (平均 $0.27 \mathrm{ng} / \mathrm{L}$ ) ; HCB 浓度为 $\mathrm{ND} \sim$ $0.72 \mathrm{ng} / \mathrm{L}$ (平均为 $0.11 \mathrm{ng} / \mathrm{L}$ ) ; $\Sigma \mathrm{HCH}_{4}$ 浓度为 $0.24 \sim 22.0 \mathrm{ng} / \mathrm{L}$ (平均为 $7.4 \mathrm{ng} / \mathrm{L}$ ), 其中 $\beta-H C H$ 浓度最高 (平 均 $3.9 \mathrm{ng} / \mathrm{L}$ ), 占 $\Sigma \mathrm{HCH}_{4}$ 的 $52.6 \%$, 其次是 $\delta-\mathrm{HCH}$ (平均为 $2.1 \mathrm{ng} / \mathrm{L}$ ) 和 $\alpha-\mathrm{HCH}$ (平均为 $1.1 \mathrm{ng} / \mathrm{L}$ ), 分别占 $\Sigma \mathrm{HCH}_{4}$ 的 $28.1 \%$ 和 $15.2 \%, \gamma-\mathrm{HCH}$ 浓度最低, 占 $4.1 \%$, 由此可见, 云南湖泊水体中主要存在的有机氯农药为 $\mathrm{HCHs}^{[18]}$, 与云贵高原湖区其它湖泊水体浓度相比处于较高污染水平, 与其它湖区水体浓度相比处于中等 污染水平. 由此可见, 中国湖泊水体中的有机氯农药主要以 HCHs 与 DDX 为主, 其中 $\mathrm{HCH}$ 单体以 $\beta-\mathrm{HCH}$ 为 主; 各湖区间 $\Sigma \mathrm{HCH}_{4}$ 浓度相差不大, 而 $\Sigma \mathrm{DDX}$ 浓度相差较大, 其中以东部湖区湖泊水体中 $\Sigma \mathrm{DDX}$ 浓度最高, 青藏一新疆湖区水体中 $\Sigma$ DDX 最低.

\section{3 中国湖泊水体中其它 POPs 的污染现状}

除上述 PAHs, OCPs 两类常见有机污染物外, PCBs、PAEs、PCDEs、PBDEs 以及 PFOSs 和 PFOAs 等污染 物在中国湖泊水体中的分布状况也有少量研究, 已有研究的湖泊数量依次为 $4 、 6 、 1 、 1$ 及 2 , 如表 1 所示. $\mathrm{Li}$ 等 2003 年 7 月研究了白洋淀 10 种 PCBs 浓度状况, 发现其浓度范围为 $0.11 \sim 2.4 \mathrm{ng} / \mathrm{L}^{[14]}$; Dai 等 2008 年 7 月 
表 1 其它种类 POPs 在中国湖库水体中的 平均浓度

Tab. 1 The average concentrations of other types of POPs in water of Chinese lakes and reservoirs

\begin{tabular}{llc}
\hline POPs 种类 & 湖泊名称 & 平均浓度 $/(\mathrm{ng} / \mathrm{L})$ \\
\hline PCBs & 白洋淀 & $23.1 \pm 22.2$ \\
& 东湖 & $2.7 \pm 0.14$ \\
& 红枫湖 & $1.4 \pm 0.1$ \\
& 三峡水库 & $0.19 \pm 0.12$ \\
PAEs & 月湖 & $724.6 \pm 36.2$ \\
& 三峡水库 & $848.1 \pm 42.4$ \\
& 北湖 & $8358.7 \pm 3385.4$ \\
& 南湖 & $6690.0 \pm 1756.4$ \\
& 雁鸣湖 & $6283.3 \pm 1515.8$ \\
PCDEs & 八一水库 & $6680.0 \pm 3455.1$ \\
PBDEs & 巢湖 & $1.1 \pm 0.06$ \\
PFOSs & 白洋淀 & $1.9 \pm 2.2$ \\
& 太湖 & $15.8 \pm 5.8$ \\
PFOAs & 太巢湖 & $27.8 \pm 20.4 *$ \\
& 太湖 & $55.8 \pm 29.4$ \\
\hline
\end{tabular}

*为除去两个工业企业旁采样点.
测定了白洋淀水体 PCBs 浓度, 发现其总浓度范围为 $19.5 \sim 131.6 \mathrm{ng} / \mathrm{L}$, 平均浓度为 $45.4 \pm 25.4 \mathrm{ng} / \mathrm{L}(n=$ $15)^{[13]}$, 高于 $\mathrm{Li}$ 等此前的结果. Zhang 等 2015 年 6 月 25 日至 7 月 10 日采集了巢湖 10 个采样点水样并测定 了其中 15 种 PCDEs 浓度, 其总浓度范围为 $0.35 \sim 2.0$ $\mathrm{ng} / \mathrm{L}$, 平均浓度为 $1.1 \mathrm{ng} / \mathrm{L}(n=10)^{[19]}$. 张蓉等于 2011 年 7 月采集了巢湖 40 个样点水样, 检测了其中 PFOSs 浓度, 发现其 PFOSs 浓度范围为 8.4 106 ng/L( 此为 除去 2 个有工业企业的采样点的数值范围, 有工业企 业的湖域 PFOSs 浓度可高达 $400 \mathrm{ng} / \mathrm{L})$, 平均浓度为 $27.8 \pm 20.4 \mathrm{ng} / \mathrm{L}(n=38$, 除去两个工业企业旁样 点) ${ }^{[20]}$. Chen 等 2012 年 5 月在太湖设置了 22 个采样 点, 测定了水样中全氟烷基物质及其异构体浓度, 发现 PFOSs 浓度范围为 $2.3 \sim 18.3 \mathrm{ng} / \mathrm{L}$ (平均浓度为 $11.0 \pm$ $3.8 \mathrm{ng} / \mathrm{L}(n=22))$, 低于此前张蓉等在巢湖的检测数 值, PFOAs 浓度范围为 $7.9 \sim 34.5 \mathrm{ng} / \mathrm{L}$ ( 平均浓度为 $24.8 \pm 6.3 \mathrm{ng} / \mathrm{L}), \sum$ PFASs 浓度范围为 $17.2 \sim 94.4 \mathrm{ng} / \mathrm{L}$ (平均浓度为 $64.7 \pm 17.8 \mathrm{ng} / \mathrm{L})^{[21]}$. 高秋生等 2016 年 3 月在白洋淀采集了 15 个采样点水样, 发现水体中 10 种 PBDEs 总浓度范围为 $0 \sim 6.5 \mathrm{ng} / \mathrm{L}$, 平均浓度为 $1.9 \pm 2.2$ $\mathrm{ng} / \mathrm{L}(n=15)$, 组成以 BDE-2 为主 $(65.8 \%)$, 可能主要

来源于大气远距离传输和高溴代联苯醚的降解 ${ }^{[5]}$. 宋慧婷等 2006 年 4 月在武汉月湖设置了 8 个采样点, 采 集水样并对其中 6 种 PAEs 浓度进行了测定, 发现其 $\Sigma$ PAE 6 浓度范围为 441.9 917.1 ng/L, 平均浓度为 $724.6 \mathrm{ng} / \mathrm{L}^{[22]}$, 远低于黄晓丽等 2016 年在长春市 4 个湖泊所测定的水体 $\Sigma P A E s$ 浓度 ${ }^{[23]}$.

\section{3 中国湖泊水体中 POPs 的分布特征}

\section{1 湖泊水体 POPs 浓度与季节的关系}

在同一湖泊相同采样点水体中 POPs 的浓度往往会因季节不同而存在差异. 于英鹏等 2012 年 3 月和 6 月在太湖 7 个采样点分别采集水样并对其中的 PAHs 进行了分析, 发现 3 月 $\Sigma \mathrm{PAH}_{16}$ 浓度范围为 63.5 393.9 $\mathrm{ng} / \mathrm{L}$, 平均浓度为 $187.8 \pm 108.7 \mathrm{ng} / \mathrm{L}(n=7), 6$ 月 $\Sigma \mathrm{PAH}_{16}$ 浓度范围为 $21.1 \sim 64.6 \mathrm{ng} / \mathrm{L}$, 平均浓度为 $37.7 \pm 16.0$ $\mathrm{ng} / \mathrm{L}(n=7)$, 从季节分布特征看, 所有采样点 $\Sigma \mathrm{PAH}_{16}$ 浓度均为 3 月高于 6 月, 可能是由于 6 月降水相对于 3 月而言较多, 地表径流的增加稀释了湖泊水体, 导致 $\Sigma \mathrm{PAH}_{16}$ 浓度降低 ${ }^{[24]}$. Tao 等于 2015 年 3 月- 2016 年 1 月中 3 个季度 (春季: 2015 年 3 月 30 日- 4 月 1 日、夏季: 2015 年 9 月 6 日- 8 日和冬季: 2016 年 1 月 27 日 和 28 日) 分别在太湖设立 $23 、 20$ 和 15 个采样点, 测定了其水样中 PAHs 浓度, 发现春季 $\Sigma \mathrm{PAH}_{16}$ 平均浓度为 $474.0 \pm 37.8 \mathrm{ng} / \mathrm{L}(n=23)$, 夏季为 $195.9 \pm 40.9 \mathrm{ng} / \mathrm{L}(n=20)$, 冬季为 $212.7 \pm 40.6 \mathrm{ng} / \mathrm{L}(n=15)$, 呈现出春季> 冬季>夏季的趋势, 其原因是各季节 PAHs 水一气界面交换量不同以及春季浮游植物量增加,这些浮游生物 从表层沉积物聚集到水体中, 导致水体中溶解态 $\mathrm{PAHs}$ 浓度增加, 从而使 $\Sigma \mathrm{PAH}_{16}$ 浓度上升; 而在冬季时, 浮 游植物沉降, 水体中 $\Sigma \mathrm{PAH}_{16}$ 浓度随之降低 ${ }^{[25]}$, 其中春季与夏季 $\Sigma \mathrm{PAH}_{16}$ 浓度高于此前于英鹏等测定同一水 体中 $\Sigma \mathrm{PAH}_{16}$ 的浓度 $187.8 \pm 108.7 \mathrm{ng} / \mathrm{L}(n=7) 、 37.7 \pm 16.0 \mathrm{ng} / \mathrm{L}(n=7)$. 唐访良等在千岛湖夏季丰水期 ( 2011 年 7 月) 和冬季枯水期 (2011 年 11 月) 分别采集了 12 个采样点水样, 测定了水样中 10 种 OCPs 浓度, 发现夏 季时, 库区 7 个采样点位有 4 个采样点检出 $\mathrm{OCPs}$, 检出率为 $57.1 \%, \Sigma O C \mathrm{CP}_{10}$ 浓度范围为 $2.0 \sim 7.6 \mathrm{ng} / \mathrm{L}$ (平均 浓度为 $3.6 \mathrm{ng} / \mathrm{L}(n=4)$ ), 其中 $\Sigma \mathrm{HCH}_{4}$ 浓度平均浓度为 $1.0 \mathrm{ng} / \mathrm{L}$, $\Sigma \mathrm{DDX}$ (包括 $p, p^{\prime}$-DDE、 $p, p^{\prime}-\mathrm{DDD} 、 o, p^{\prime}-$ $\mathrm{DDT}, p, p^{\prime}$-DDT) 平均浓度为 $3.6 \mathrm{ng} / \mathrm{L}$, 枯水期所有采样点位均有 $\mathrm{OCPs}$ 被检出, 库区水体 $\Sigma O C \mathrm{CP}_{10}$ 浓度范围为 $1.9 \sim 6.3 \mathrm{ng} / \mathrm{L}$ (平均浓度为 $3.7 \mathrm{ng} / \mathrm{L}(n=7)$ ), 其中 $\Sigma \mathrm{HCH}_{4}$ 平均浓度为 $0.52 \mathrm{ng} / \mathrm{L}, \Sigma \mathrm{DDX}$ 平均浓度为 $3.3 \mathrm{ng} /$ 
$\mathrm{L}$; 从时间分布上看, 丰水期 ( 7 月 ) $\Sigma O C \mathrm{PP}_{10}$ 浓度略低于枯水期 (11 月), 而丰水期 $\Sigma H_{C H}$ 与 $\Sigma \mathrm{DDX}$ 浓度则略 大于枯水期浓度 ${ }^{[26]}$. 黄晓丽等于 2016 年 5-7 月在长春市四 4 个湖泊: 北湖、南湖、雁鸣湖和八一水库各设 立了 3 个采样点, 检测了湖泊水体中 8 种邻苯二甲酸酯 (PAEs) 的浓度, 发现北湖 5-7 月 $\Sigma \mathrm{PAE}_{8}$ 平均浓度 依次为 $13.1 、 6.6$ 和 $5.0 、 39.0 \mathrm{ng} / \mathrm{mL}$; 南湖依次为 $8.8 、 6.8$ 和 $4.5 \mathrm{ng} / \mathrm{mL}$, 雁鸣湖依次为 $7.4 、 7.3$ 和 $4.1 \mathrm{ng} / \mathrm{mL}$, 八一水库依次为 $5.6 、 3.1$ 和 $11.4 \mathrm{ng} / \mathrm{mL}$, 表明除八一水库外, 其它 3 个湖泊 $\Sigma \mathrm{PAE}_{8}$ 的浓度均是 5 月 $>6$ 月 $>7$ 月,其原因是 7 月份降雨量高于 $5 、 6$ 月, 地表径流增加汇人湖泊, 对水体进行了稀释所致 ${ }^{[23]}$. 这些研究均说 明湖泊水体中 POPs 的浓度与季节有关, 且夏季湖泊水体中 POPs 浓度往往低于其它季节 (丰水期 <枯水 期). 分析其原因, 可能与降水 (中国夏季降水量相较于其它季节最大)、地表径流量 (地表径流携带陆地上 的 POPs 进人湖泊水体并对其进行了稀释)、浮游植物生物量 (影响 POPs 在水中的自由溶解态浓度) 、水一气 界面交换量、污染源等因素有关.

\section{2 湖泊水体 POPs 浓度与水体深度的关系}

中国湖泊水体中 POPs 的浓度还与所处水体的深度有关. 鲁玥于 2010 年 5 月和 9 月分别在太湖采集了 30 个和 11 个采样点的水样, 并分析了上覆水和间隙水样品中 POPs 浓度 (图 3), 发现 5 月份上覆水中 $\Sigma \mathrm{PAH}_{16}$ 浓度范围为 $238 \sim 7422 \mathrm{ng} / \mathrm{L}$ (平均浓度为 $1592 \mathrm{ng} / \mathrm{L}(n=30)$ ), $\Sigma O C \mathrm{P}_{9}$ 浓度范围为 $24.3 \sim 155.6 \mathrm{ng} / \mathrm{L}$ (平均浓度为 $95.2 \mathrm{ng} / \mathrm{L}$ ), $\Sigma \mathrm{DDDX}$ 浓度范围为 $23.6 \sim 151.9 \mathrm{ng} / \mathrm{L}$ (平均浓度为 $92.4 \mathrm{ng} / \mathrm{L}$ ) ; 间隙水中 $\Sigma \mathrm{PAH}_{16}$ 浓 度范围为 $2012 \sim 19899 \mathrm{ng} / \mathrm{L}$ (平均浓度为 $4247 \mathrm{ng} / \mathrm{L}$ ), $\Sigma O C \mathrm{CP}_{9}$ 浓度范围为 $137.0 \sim 2192.5 \mathrm{ng} / \mathrm{L}$ (平均浓度为 $581.4 \mathrm{ng} / \mathrm{L}$ ), $\sum D D X$ 浓度范围为 134.2 2157.1 ng/L(平均浓度为 $567 \mathrm{ng} / \mathrm{L}$ ); 间隙水中各 POPs 浓度均大于 上覆水. 9 月上覆水中 $\Sigma \mathrm{PAH}_{16}$ 浓度范围为 $1230 \sim 5034 \mathrm{ng} / \mathrm{L}$ (平均浓度为 $3030 \mathrm{ng} / \mathrm{L}(n=11)$ ), $\Sigma O C \mathrm{P}_{9}$ 范围为 95.0 721.2 ng/L(平均浓度为 $342.1 \mathrm{ng} / \mathrm{L}$ ), $\Sigma \mathrm{DDX}$ 浓度范围为 $58.5 \sim 217.6 \mathrm{ng} / \mathrm{L}($ 平均浓度为 $101.6 \mathrm{ng} / \mathrm{L}$ ); 间隙水中 $\Sigma \mathrm{PAH}_{16}$ 浓度范围为 $1855 \sim 9847 \mathrm{ng} / \mathrm{L}$ (平均浓度为 $5472 \mathrm{ng} / \mathrm{L}$ ), $\Sigma O C \mathrm{CP}_{9}$ 浓度范围为 $124.8 \sim 700.1$ $\mathrm{ng} / \mathrm{L}$ (平均浓度为 $420.8 \mathrm{ng} / \mathrm{L}$ ), $\Sigma \mathrm{DDDX}$ 浓度范围为 $124.8 \sim 700.1 \mathrm{ng} / \mathrm{L}$ (平均浓度为 $417.2 \mathrm{ng} / \mathrm{L}$ ) ${ }^{[27]}$, 同 5 月研 究结论相同, 即间隙水中各 POPs 浓度均大于上覆水中浓度. 吴敏于 2012 年 12 月采集丹江口水库 19 个采 样点的表面水与孔隙水水体, 测试了其中 20 种 OCPs 浓度, 共检测出 11 种 OCPs, 发现表面水体中 $\Sigma O C P_{20}$ 的 平均浓度为 $2.3 \mathrm{ng} / \mathrm{L}$, 孔隙水中 $\Sigma O C \mathrm{CP}_{20}$ 的平均浓度为 $5.5 \mathrm{ng} / \mathrm{L}$, 其中 $\Sigma \mathrm{HCH}_{4}$ 浓度范围为 $12.7 \sim 36.6 \mathrm{ng} / \mathrm{L}$, $\sum \mathrm{DDX}$ (包括 $p, p^{\prime}$-DDE, $p, p^{\prime}$-DDD, $o, p^{\prime}$-DDT, $p, p^{\prime}$-DDT) 浓度范围为 $0 \sim 517.5 \mathrm{ng} / \mathrm{L}$, 表面水中被检出的有机 氯农药以 DDX 为主, $\Sigma D D X$ 浓度占 $\Sigma O C P_{20}$ 总浓度的 $97.4 \%$; 但在孔隙水中, 被检出的有机氯农药则以 $\mathrm{HCHs}$ 为主, $\Sigma \mathrm{HCH}_{4}$ 浓度占 $\Sigma O C \mathrm{CP}_{20}$ 总浓度的 $49.7 \%{ }^{[28]}$. 曲明昕研究了百花湖 4 个采样点 (共 8 个样品) 表层水和 底层界面水中 $\Sigma \mathrm{PAH}_{16}$ 浓度, 发现表层水中 $\Sigma \mathrm{PAH}_{16}$ 浓度范围为 $421.3 \sim 515.0 \mathrm{ng} / \mathrm{L}$ (平均浓度为 $475.1 \mathrm{ng} / \mathrm{L}$ $(n=4))$, 底层界面水中 $\sum \mathrm{PAH}_{16}$ 浓度范围为 $687.9 \sim 760.6 \mathrm{ng} / \mathrm{L}($ 平均浓度为 $726.3 \mathrm{ng} / \mathrm{L}(n=4))$, 底层界面 水中 $\Sigma \mathrm{PAH}_{16}$ 浓度高于表层水中浓度 ${ }^{[29]}$. 通过对这些研究结果的分析, 可以发现中国湖泊水体中间隙水 POPs 浓度高于上覆水中的浓度, 分析其原因可能是水体中 POPs 易附着在悬浮颗粒物及浮游植物上, 可随 沉降作用进人湖泊沉积物中, 而间隙水相较于表层上覆水而言距沉积物更近, 因此沉积物中的 POPs 溶解进 人间隙水导致其中 POPs 浓度高于表层水.

\section{3 湖泊水体 POPs 浓度与采样点位置的关系}

同一湖泊中各点位水体 POPs 浓度也会受水体流向、所处位置的影响. 李红莉等于 2002 年 8 月和 11 月 在南四湖湖区及主要人湖河口处取水样, 对其中多环芳烃浓度进行检测, 发现湖内采样点水体 $\Sigma \mathrm{PAH}_{16}$ 浓度 范围为 $523 \sim 3530 \mathrm{ng} / \mathrm{L}$, 平均浓度为 $1564.5 \pm 1004.9 \mathrm{ng} / \mathrm{L}(n=6)$; 而人湖河口采样点水体 $\Sigma \mathrm{PAH}_{16}$ 浓度范围为 $4 \sim 2200 \mathrm{ng} / \mathrm{L}$, 平均浓度为 $468.9 \pm 775.5 \mathrm{ng} / \mathrm{L}(n=7)$, 从空间分布来看, 人湖河口采样点水体 $\Sigma \mathrm{PAH}_{16}$ 的浓度 低于湖内采样点, 湖内各采样点 $\Sigma \mathrm{PAH}_{16}$ 浓度随水流方向逐渐增大 ${ }^{[30]}$. 梁一灵等于 2007 年 6 月底在杭州西 湖的 7 个采样点采集了水样, 测定了水样中 17 种 OCPs 的浓度, 发现人水口两个采样点水体 $\Sigma O C P_{17}$ 浓度范 围为 8.0 45.1 ng/L, 平均浓度为 $26.6 \pm 18.5 \mathrm{ng} / \mathrm{L}(n=2)$, 湖心处 $\Sigma O C P_{17}$ 浓度为 $36.0 \mathrm{ng} / \mathrm{L}$, 排水口采样点水 体 $\Sigma O C P_{17}$ 浓度范围为 $44.7 \sim 69.5 \mathrm{ng} / \mathrm{L}$, 平均浓度为 $57.1 \pm 12.4(n=2)$, 水体各区域 $\Sigma O C P_{17}$ 浓度呈现出人水 口 <湖心 <排水口的趋势. 由此分析得出, 西湖水域中 OCPs 浓度随水流方向逐渐升高 ${ }^{[31]}$. 吕海波于 2013 年 2 月和 8 月分别在锦绣川水库采集了 8 个和 12 个采样点的水样, 测定了水样中的 17 种 OCPs 浓度, 发现 


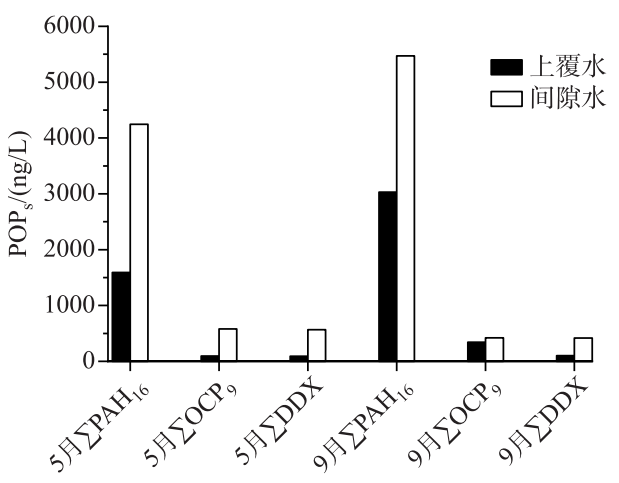

图 3 太湖水体 2010 年 5 月和 9 月各 POPs 平均浓度 (修改自文献 [27]) $\left(\Sigma \mathrm{PAH}_{16}\right.$ 为 16 种优先控制污染物; $\Sigma \mathrm{OCP}_{9}$ 为检测的 9 种有机氯农药浓度之和; $\Sigma D D X$ 为 DDE、DDD 和 DDT 浓度之和)

Fig. 3 The average concentrations of POPs in the water of Lake Taihu in May and September of 2010
2013 年 2 月时, 水库进水口水体 $\Sigma O C P_{17}$ 最低浓度为 1.6 $\mathrm{ng} / \mathrm{L}$, 从湖泊上游至下游各采样点水体 OCPs 的浓度呈 上升趋势, 出水口 $\Sigma O C P_{17}$ 浓度最高达 $5.3 \mathrm{ng} / \mathrm{L} ; 2013$ 年 8 月增加到 12 个采样点, 发现水库进水口水体 $\Sigma \mathrm{OCP}_{17}$ 浓度为 $0.57 \mathrm{ng} / \mathrm{L}$, 出水口水体 $\Sigma O C P_{17}$ 浓度为 $0.68 \mathrm{ng} / \mathrm{L}$, 整体上看水体 $\Sigma O C P_{17}$ 浓度差异不大, 出水口水体略高于 进水口水体 ${ }^{[32]}$. 上述研究表明中国湖泊水体中不同采样 点水体 POPs 浓度高低顺序多数呈现出人湖河口 <湖心 (<排水口), 且湖内各采样点 POPs 浓度随水流方向逐渐 升高, 说明 POPs 会随水体运动发生物理迁移并在下一 级区域水体积累, 导致该区域水体较上一级浓度升高.

\section{4 湖泊水体 POPs 浓度与人为活动影响的关系}

王元等于 2014 年春 $(4-5$ 月)、夏 $(7-8$ 月)、秋 (9-10月)、冬( $12-2015$ 年 1 月) 在巢湖湖区及人湖河 流共 40 个样点进行了季节性水样采样, 对水体中的 22 种 OCPs 进行了检测, 发现在所有采样点水样中, 最多只 有六六六类农药 $(\mathrm{HCHs}$ ) 、六氯苯、七氯、艾氏剂、硫丹 I 以及环氧七氯等 10 种目标 OCPs 被检出. 此结果与中国 此前大量生产与使用的农药种类有关, 此类农药喷酒灌溉之后, 可能会因干湿沉降过程或随地表径流而污 染周边土壤以及河流湖泊, 产生环境残留; 除此之外, 与秋冬季相比, 春夏两季表层水样中少一种顺式氯丹 目标物的检出, 可能因在秋冬两季, 当地人使用了较多的顺式氯丹农药进行杀虫, 从而导致水体 OCPs 组成 的差异 ${ }^{[33]}$. 梁一灵等于 2007 年 6 月底采集了杭州西湖 7 个区域水样, 检测了其中 17 种 OCPs 浓度, 发现浴 鹄湾 $(112.2 \mathrm{ng} / \mathrm{L})$ 与茅家埠 $(126.2 \mathrm{ng} / \mathrm{L})$ 两个区域 $\Sigma O C P_{17}$ 浓度远高于其它 5 个区域浓度 $\left(\Sigma O C P_{17}\right.$ 平均浓度 为 $40.7 \pm 19.8 \mathrm{ng} / \mathrm{L})$, 结合两点地理位置及周边环境, 发现这两个采样点位于西湖西南部和西部, 附近有山溪 和少量赤山埠水厂的人水, 且由于周边地区过去长期作为农业用地耕作, 有大量的农药残留于土壤中, 可能 在雨水和溪流的冲刷下随地表径流汇集于此, 因而残留有机氯农药也最多; 另外圣塘闸 $(69.5 \mathrm{ng} / \mathrm{L})$ 是西湖 最大的排水口, 位于西湖的东部和东北部, 是西湖主要游船码头驻地, 游人的大量活动和船只往来会对湖底 污泥产生扰动, 加剧污染物的释放, 这可能是导致圣塘闸水样 OCPs 浓度略高于其它 4 个区域的原因 ${ }^{[31]}$. 张 蓉等检测了巢湖 40 个采样点水样中的 PFOS 浓度, 发现水体中 PFOS 浓度大都处在 $8.4 \pm 1.2$ 和 $106 \pm 10.2$ $\mathrm{ng} / \mathrm{L}$ 之间, 而皖维排污口和裕溪闸口两处水样中的 PFOS 浓度分别高达 $400 \pm 51 、 350 \pm 45 \mathrm{ng} / \mathrm{L}$, 其它样点中, 流经巢湖市居民区的支流天河水样中 PFOS 的浓度较高 $(70 \mathrm{ng} / \mathrm{L})^{[20]}$, 说明人类生产生活所产生的污染物 是导致巢湖水体 PFOS 浓度较高的主要原因. 昌盛等研究分析了 2014 年 5 月在于桥水库库区 6 个采样点水 样中的 PAHs 浓度, 发现水体中高环 PAHs 和低环 PAHs 浓度相当, 2 3 环 $\Sigma$ PAHs 与 4 6 环 $\Sigma$ PAHs 平均浓 度分别为 $287.1 、 281.5 \mathrm{ng} / \mathrm{L}^{[34]}$, 而低环数 PAHs 主要来源为石油制品及燃油, 高环数 $\mathrm{PAHs}$ 主要来自煤和生 物质能源的燃烧 ${ }^{[35-36]}$, 由此推断于桥水库库区 PAHs 受周边村庄生活燃煤与生物质影响较大. 通过上述研 究发现, 人类活动对湖泊水体中 POPs 浓度及分布特征存在较大影响, 不论是工厂排污、农药喷酒、航道行 船,还是居民生活污水的排放,都会使周边湖泊水体中 POPs 浓度升高或改变其原本的分布特征.

\section{5 湖泊水体 POPs 浓度与自身理化性质的关系}

同一湖泊或水库水体中不同种类 POPs 浓度会因自身理化性质不同而存在差异. 例如上文提到的湖泊 水体中低分子量 PAHs 浓度普遍高于高分子量 PAHs 浓度. 可能存在两种原因: 一是高分子量 PAHs 溶解度 较小, 低分子量 PAHs 溶解度较大; 二是高分子量的 PAHs 疏水性、亲脂性更强, 更易于被沉积物、悬浮颗粒 物及生物吸附或富集.

已有的研究表明 $\beta-\mathrm{HCH}$ 是中国湖泊水体中 $\mathrm{HCH}$ 的优势异构体. 一方面是由于 $\beta-H C H$ 水溶性和挥发性 较低 ${ }^{[7]}$, 且具有良好的对称性, 使其物化性质相较于其它异构体更为稳定、不易降解; 另一方面, 其它不稳定 
$\mathrm{HCH}$ 异构体经过长时间的迁移转化, 可以转变为 $\beta-\mathrm{HCH}$ 以达到稳定状态 ${ }^{[37]}$. 这些因素共同作用, 导致了湖 泊水体中 $\beta-\mathrm{HCH}$ 浓度相对较高.

DDX 化学性质稳定, 在常温条件下不易发生分解 ${ }^{[38]}$ 且能在大气中远距离迁移并能再次沉降至地表 ${ }^{[39]}$, 因此高原湖泊水体中 DDX 的分布可能还受到农业较为发达地区 DDX 迁移的影响.

PCBs 同系物在中国湖泊和水库水体中的浓度与其氯取代的数目有关. 习志群等 ${ }^{[40]}$ 研究发现氯取代数 量的不同会造成 PCBs 同系物的物理化学性质的不同, 低氯取代的 PCBs 水溶性大于高氯取代的 PCBs. 因此 在水体中, 低氯取代的 PCBs 大都存在于水体中, 而高氯取代的 PCBs 更易于富集在沉积物中, 从而导致湖泊 水体中低氯取代的 PCBs 检出浓度高于高氯取代的 PCBs. 另外, 由于 PCBs、PAEs、PBDEs 等水溶性较差且均 具有较大的疏水性 ${ }^{[41-43]}$, 使其在水体中易附着于悬浮颗粒物上, 最终沉降进人沉积物中, 因此湖泊水体中 PCBs、PAEs、PBDEs 等 POPs 浓度往往低于沉积物中, 且其在表层水体中的浓度通常低于间隙水中的浓度.

\section{4 中国湖泊水体中不同种类 POPs 浓度的变化趋势及差异}

根据所收集到的 80 篇公开发表文献,发现中国湖泊水体中不同种类 POPs 的浓度不同,截止 2019 年 2 月已有的研究数据中, $\Sigma \mathrm{PAH}_{16}$ 平均浓度为 $360.0 \pm 433.8 \mathrm{ng} / \mathrm{L}(n=26), \Sigma \mathrm{HCH}_{4}$ 平均浓度为 $12.8 \pm 23.5 \mathrm{ng} / \mathrm{L}$ $(n=30), \Sigma \mathrm{DDX}$ 平均浓度为 $12.8 \pm 24.6 \mathrm{ng} / \mathrm{L}(n=24), \Sigma \mathrm{PAH}_{16}$ 浓度高于 $\Sigma \mathrm{HCH}_{4}$ 和 $\Sigma \mathrm{DDX}$, 这是因为 $\mathrm{PAHs}$ 来 源广泛, 主要来源包括自然源和人为源: 其中自然源主要包括燃烧 (森林大火和火山喷发) 和生物合成 (沉积 物成岩过程、生物转化过程和焦油矿坑内气体), 未开采的煤、石油中也含有大量的 PAHs; 人为源来自于工 业工艺过程中的三废排放、欠氧燃烧 (燃油锅炉、窑炉、汽车、轮船、内燃机车等)、垃圾堆集与填埋及直接的 交通排放以及伴随的轮胎磨损、路面磨损产生的沥青颗粒以及道路扬尘 ${ }^{[44]}$, 这些 PAHs 产生源与人类生产 生活息息相关,无法进行严格意义上的控制,而 HCHs、DDX 等大部分有机氯农药目前在中国已被禁止生产 和使用, 因此其浓度与 $\Sigma \mathrm{PAH}_{16}$ 相比较低. 从时间上看, 2010 年及以前, $\Sigma \mathrm{PAH}_{16} 、 \Sigma \mathrm{HCH}_{4}$ 及 $\Sigma \mathrm{DDX}$ 的平均浓度 分别为 $537.7 \pm 729.9 \mathrm{ng} / \mathrm{L}(n=13) 、 20.1 \pm 32.2 \mathrm{ng} / \mathrm{L}(n=24)$ 和 $21.3 \pm 31.8 \mathrm{ng} / \mathrm{L}(n=17) ; 2010$ 年后, $\Sigma \mathrm{PAH}$ 、 $\Sigma \mathrm{HCH}_{4}$ 和 $\Sigma \mathrm{DDX}$ 的平均浓度分别为 $322.5 \pm 340.0 \mathrm{ng} / \mathrm{L}(n=20) 、 11.0 \pm 12.2 \mathrm{ng} / \mathrm{L}(n=7)$ 和 $10.7 \pm 18.7 \mathrm{ng} / \mathrm{L}(n=$ 8) (图 4).

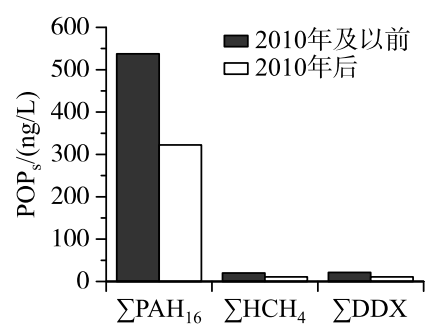

(a)

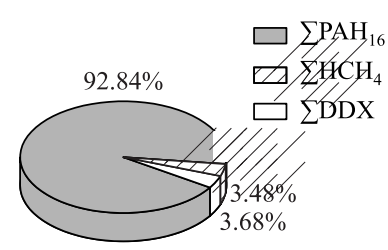

(b)

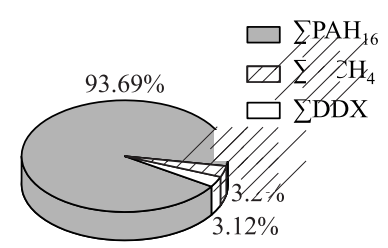

(c)

图 4 中国各湖泊、水库水体中 $\Sigma \mathrm{PAH}_{16} 、 \Sigma \mathrm{HCH}_{4}$ 和 $\Sigma \mathrm{DDX}$ 在 2010 年前后 平均浓度对比 $(\mathrm{a})^{[5-11,13-18,22,24,25,27,28,32,34,49-63,66-71,73-77,78-83,84-99]}, 2010$ 年及以前 $\Sigma \mathrm{PAH}_{16}$ 、 $\Sigma \mathrm{HCH}_{4}$ 和 $\Sigma \mathrm{DDX}$ 相对比例 (b) ${ }^{[6,9,10,13-15,17,18,20,22,27,29-31,49-53,57,59,63-70,72-78,81,83,87,89,90,96,97,99]}$, 2010 年后 $\Sigma \mathrm{PAH}_{16} 、 \Sigma \mathrm{HCH}_{4}$ 和 $\Sigma \mathrm{DDX}$ 相对比例 $(\mathrm{c})^{[5,7,8,11,16,19,21,23-26,28,32-34,54-56,58,60-62,71,79,80,82,84-86,88,91-95,98]}$

Fig.4 The comparison of $\Sigma \mathrm{PAH}_{16}, \Sigma \mathrm{HCH}_{4}$ and $\Sigma \mathrm{DDX}$ in water of Chinese lakes and reservoirs before and after 2010 (a), relative proportions of $\Sigma \mathrm{PAH}_{16}, \Sigma \mathrm{HCH}_{4}$ and $\Sigma \mathrm{DDX}$ in 2010 and before (b), and after 2010 (c)

湖泊水体中 $\Sigma \mathrm{PAH}_{16} 、 \Sigma \mathrm{HCH}_{4}$ 和 $\Sigma \mathrm{DDX}$ 相对总比例无显著变化,但 2010 年后这三类 POPs 浓度较 2010 年及以前都有所降低. 中国于 2001 年签署了《关于持久性有机污染物 (POPs) 的斯德哥尔摩公约》(Stockholm convention on persistent organic pollutants), 该公约于 2004 年正式生效 ${ }^{[45]}$, 至 2011 年, 该公约中受控制 的 POPs 增至 22 种,包括 HCHs、DDX、PCBs 等. 中国宣布自 2009 年 5 月 17 日起,禁止在境内生产、流通、使 用和出口滴滴涕、六氯苯等 ${ }^{[46]}$, 从本文收集到的数据来看, 中国履行《关于持久性有机污染物 (POPs) 的斯 
德哥尔摩公约》以及对 PAHs 的管控减排已显成效.

\section{5 结论}

收集整理了截止到 2019 年 2 月公开发表的关于中国湖泊水体 POPs 的 80 篇文献数据, 涉及中国 19 个 省、直辖市、自治区的 49 个湖泊, 系统地总结了中国湖泊水体 POPs 浓度的污染现状及分布特征, 发现: 中国 湖泊水体受到了不同程度的 POPs 污染, 其中 PAHs、HCHs 和 DDX 是研究最多的三类污染物, 其总平均浓度 分别为 $360.0 \pm 433.8 \mathrm{ng} / \mathrm{L}(n=26) 、 12.8 \pm 23.5 \mathrm{ng} / \mathrm{L}(n=30) 、 12.8 \pm 24.6 \mathrm{ng} / \mathrm{L}(n=24) ; \mathrm{PAHs}$ 在不同湖泊水体 中的浓度差异较大, 而 DDX 和 HCHs 浓度在不同湖泊水体中差异相对较小; 在空间分布上, 中国湖泊水体 POPs 的研究多集中在东部平原地区 $(n=32)$, 云贵高原湖区 $(n=14)$ 和西藏高原湖区 $(n=3)$ 有少量研究, 而 其它地区基本没有研究, 其中, 东部地区湖泊水体中 $\Sigma \mathrm{PAH}_{16}$ 浓度为 $379.2 \pm 461.0 \mathrm{ng} / \mathrm{L}(n=22)$, 云贵高原湖 区水体 $\Sigma \mathrm{PAH}_{16}$ 浓度为 $303.6 \pm 216.6 \mathrm{ng} / \mathrm{L}(n=3)$, 青藏一新疆湖区水体 $\Sigma \mathrm{PAH}_{16}$ 为 $189.7 \pm 9.5 \mathrm{ng} / \mathrm{L}(n=1)$, 呈 现出东部湖区>云贵高原湖区> 青藏一新疆湖区的分布特征; 东部地区湖泊水体中 $\Sigma \mathrm{HCH}_{4}$ 的浓度为 $19.6 \pm$ $32.6 \mathrm{ng} / \mathrm{L}(n=14)$, 云贵高原湖区水体 $\Sigma \mathrm{HCH}_{4}$ 浓度为 $6.6 \pm 5.2 \mathrm{ng} / \mathrm{L}(n=13)$, 青藏一新疆湖区水体 $\Sigma \mathrm{HCH}_{4}$ 浓 度为 7.9 $7.3 \mathrm{ng} / \mathrm{L}(n=3)$, 呈现出东部湖区 > 青藏一新疆湖区 > 云贵高原湖区的分布特征; 东部地区湖泊水 体 $\Sigma$ DDX 浓度为 $21.2 \pm 30.8 \mathrm{ng} / \mathrm{L}(n=13)$, 云贵高原湖区水体 $\Sigma D D X$ 浓度为 $3.4 \pm 5.6 \mathrm{ng} / \mathrm{L}(n=8)$, 青藏一新疆 湖区水体 $\Sigma D D X$ 浓度为 $1.8 \pm 2.1 \mathrm{ng} / \mathrm{L}(n=3)$, 呈现出东部湖区>云贵高原湖区> 青藏一新疆湖区的分布特 征; 从时间分布看, 2010 年后在中国湖泊水体中检测出的 $\Sigma \mathrm{PAH}_{16} 、 \Sigma \mathrm{HCH}_{4}$ 和 $\Sigma \mathrm{DDX}$ 浓度比 2010 年及以前有 所降低, 近年整体呈下降趋势, 说明中国履行《关于持久性有机污染物 ( POPs) 的斯德哥尔摩公约》内容以 及对 PAHs 的管控减排已显成效; POPs 在中国湖泊水体中的浓度与季节、水体深度 (上覆水与间隙水)、采 样点位置 (人湖口、湖心及出水口) 和人为活动 (农业耕作、生活排污、工业污水及码头船只往来等) 存在一 定关系,但并非完全受这些因素影响,还与 POPs 种类、所处区域的地理位置等诸多因素有关.

中国是 POPs 的生产与使用大国 ${ }^{[47]}$, 同时是一个湖泊众多的国家 ${ }^{[48]}$, 多数湖泊在供水、渔业等方面发挥 着重要作用, 湖泊水体质量直接影响着水质与水产品的安全以及当地生态系统及人体的健康, 因此 POPs 在 中国湖泊水体中的污染现状及分布特征研究十分重要, 然而就研究现状而言, 目前缺乏对中国湖泊水体中 POPs 的全面研究, 特别是在时间、空间上对中国湖泊水体介质中 POPs 的综合研究很匮乏. 本综述有助于了 解中国湖泊水体 POPs 的污染现状及分布特征. 但仍缺乏数据, 在今后的工作中, 应对中国湖泊水体 POPs 污染现状及分布特征开展综合性研究, 以研究它们在水体中的周期性变化、生物地球化学过程及作用机理 等,为中国湖泊 POPs 的污染预防及控制提供一定的科学理论依据.

\section{6 参考文献}

[ 1 ] Wu QH. Preliminary study on the occurrence of polycyclic aromatic hydrocarbons and organochlorine pesticides in highly polluted sediments from the Pearl River (Guangzhou section) [Dissertation]. Guangzhou: Guangzhou Institute of Geochemistry, Chinese Academy of Sciences, 2003. [吴启航. 珠江(广州段) 高污染沉积物中多环芳烃和有机氯农药赋 存状态初步研究 [学位论文]. 广州: 中国科学院广州地球化学研究所, 2003.]

[ 2 ] Liu DH, Tao YQ, Zhou WZ. Research progress on the distribution and enrichment of persistent organic pollutants in Chinese lake organisms. J Lake Sci, 2018, 30(3) : 581-596. DOI: 10.18307/2018.0301. [ 刘东红, 陶玉强, 周文佐. 持久 性有机污染物在中国湖泊生物中分布与富集的研究进展. 湖泊科学, 2018, 30(3): 581-596.]

[ 3 ] Li QY. Adsorption and degradation of POPs by phytoplankton and its physiological and ecological responses [Dissertation]. Nanjing: Nanjing Normal University, 2015. [梛倩玉. 浮游藻类对 POPs 的吸附降解及其生理生态响应研究 [ 学位论 文]. 南京: 南京师范大学, 2015.]

[ 4 ] Wu QL, Xie P, Yang LY et al. The formation mechanism and prevention of ecological disasters in lake blue algae blooms. Advances in Earth Science, 2008, 23(11): 1115-1123. [ 吴庆龙, 谢平, 杨柳燕等. 湖泊蓝藻水华生态灾害形成机理 及防治的基础研究. 地球科学进展, 2008,23(11): 1115-1123.]

[ 5 ] Gao QS, Jiao LX, Yang L et al. Pollution characteristics and risk assessment of typical persistent organic pollutants in Baiyangdian. Environmental Science, 2018, 39(4) : 1616-1627. [高秋生, 焦立新, 杨柳等. 白洋淀典型持久性有机污 


\section{染物污染特征与风险评估. 环境科学, 2018, 39(4): 1616-1627.]}

[ 6 ] Guo W, Pei YS, Yang ZF et al. Assessment on the distribution and partitioning characteristics of polycyclic aromatic hydrocarbons (PAHs) in Lake Baiyangdian, a shallow freshwater lake in China. Journal of Environmental Monitoring, 2011, 13(3): 681-688.

[ 7 ] Wang YZ, Zhang J, Zhou XS et al. Seasonal pollution characteristics and source identification of polycyclic aromatic hydrocarbons and organochlorine pesticides in surface water of Baiyangdian lake. Chinese Journal of Environmental Science, 2017, 38(3) : 964-978. [王乙震, 张俊, 周绪申等. 白洋淀多环芳烃与有机氯农药季节性污染特征及来源分析. 环 境科学, 2017, 38(3) : 964-978.]

[ 8 ] Zhang JQ, Hu TP, Xing XL et al. Distribution, source and risk assessment of polycyclic aromatic hydrocarbons in surface sediments of Daye Lake. Environmental Science, 2017, 38(1): 170-179. [张家泉, 胡天鹏, 邢新丽等. 大治湖表层沉 积物-水中多环芳烃的分布、来源及风险评价. 环境科学, 2017, 38(1) : 170-179.]

[ 9 ] Qin N, He W, Kong XZ et al. Distribution, partitioning and sources of polycyclic aromatic hydrocarbons in the water-SPMsediment system of Lake Chaohu, China. Science of the Total Environment, 2014, 496: 414-423.

[10] Qin N, He W, Kong XZ et al. Atmospheric partitioning and the air-water exchange of polycyclic aromatic hydrocarbons in a large shallow Chinese lake (Lake Chaohu). Chemosphere, 2013, 93 (9): 1685-1693. DOI: 10.1016/j. chemosphere. 2013.05.038.

[11] Shen DH. Pollution investigation and ecological risk assessment on polycyclic aromatic hydrocarbons (PAHs) in Chaohu Lake [Dissertation]. Hefei : Anhui Medical University, 2014. [沈登辉. 巢湖水体中多环芳烃类化合物 (PAHs) 污染调 查和生态风险评价 [学位论文]. 合肥: 安徽医科大学, 2014.]

[12] Liu C, Chen JW. Geochemical characteristics of DDT and HCH in agricultural soil in the suburb of Beijing. Earth Science Frontiers, 2008, 15(5): 82-89. [刘晨, 陈家玮. 北京郊区农田土壤中滴滴涕和六六六地球化学特征研究. 地学前 缘, $2008,15(5): 82-89$. ]

[13] Dai GH, Liu XH, Liang G et al. Distribution of organochlorine pesticides (OCPs) and poly chlorinated biphenyls (PCBs) in surface water and sediments from Baiyangdian Lake in North China. Journal of Environmental Sciences, 2011, 23 (10): 1640-1649.

[14] Li FC, Shen YF, Wang XH et al. Monitoring organochlorine pesticides and polychlorinated biphenyls in Baiyangdian Lake using microbial communities. Journal of Freshwater Ecology, 2005, 20(4) : 751-756.

[15] Hu GC, Dai JY, Mai BX et al. Concentrations and accumulation features of organochlorine pesticides in the Baiyangdian Lake freshwater food web of North China. Archives of Environmental Contamination and Toxicology, 2010, 58 (3) : 700-710.

[16] Zhi H, Zhao ZH, Zhang L. The fate of polycyclic aromatic hydrocarbons (PAHs) and organochlorine pesticides (OCPs) in water from Poyang Lake, the largest freshwater lake in China. Chemosphere, 2015, 119: 1134-1140.

[17] Long ZY. Distribution characteristics and sources of organochlorine pesticides in Poyang Lake [Dissertation]. Nanchang: Nanchang University, 2009. [ 龙智勇. 鄱阳湖有机氯农药分布特征与来源研究 [学位论文]. 南昌: 南昌大 学, 2009.]

[18] Yang J, Zhang WJ, Shen YF et al. Monitoring of organochlorine pesticides using PFU systems in Yunnan lakes and rivers, China. Chemosphere, 2007, 66(2): 219-225.

[19] Zhang XS, Wang TT, Gao L et al. Polychlorinated diphenyl ethers (PCDEs) in surface sediments, suspended particulate matter (SPM) and surface water of Chaohu Lake, China. Environmental Pollution, 2018, 241: 441-450.

[20] Zhang R, Wang MX, Tang J et al. Preliminary analysis of PFOS pollution and drinking risk in Chaohu Lake. Journal of Anhui Agricultural University, 2012, 39(1): 92-96. [张蓉, 王明星, 唐俊等. 巢湖水体 PFOS 污染及饮用风险初步 分析. 安徽农业大学学报, 2012, 39(1): 92-96.]

[21] Chen XW, Zhu LY, Pan XY et al. Isomeric specific partitioning behaviors of perfluoroalkyl substances in water dissolved phase, suspended particulate matters and sediments in Liao River Basin and Taihu Lake, China. Water Research, 2015, 80: $235-244$.

[22] Song HT, He F, Cheng SP et al. Distribution characteristics of 25 semi-volatile organic pollutants in water and surface sediments of Yuehu Lake in Wuhan. J Lake Sci, 2010, 22(4) : 521-526. DOI: 10.18307/2010.0407. [宋慧婷, 贺锋, 成 水平等. 武汉月湖水体和表层沉积物中 25 种半挥发性有机污染物分布特性. 湖泊科学, 2010, 22(4): 521-526.] 
[23] Huang XL, Tang SZ, Qin DL et al. Composition and distribution characteristics of phthalate esters (PAEs) in urban lakes. Chinese Journal of Fisheries, 2017, 30(4) : 33-39. [黄晓丽, 汤施展, 覃东立等. 城市湖泊中邻苯二甲酸酯( PAEs) 类物质的组成及其分布特征. 水产学杂志, 2017, 30(4) : 33-39.]

[24] Yu YP, Liu M. Distribution, pollution sources, and ecological risk assessment of PAHs in water source area of Taihu Basin. Water Resources Protection, 2017, 33(3): 82-89. [于英鹏, 刘敏. 太湖流域水源地多环芳烃分布、溯源与生态风 险评估. 水资源保护, 2017, 33(3): 82-89.]

[25] Tao YQ, Yu J, Lei GL et al. Indirect influence of eutrophication on air-water exchange fluxes, sinking fluxes, and occurrence of polycyclic aromatic hydrocarbons. Water Research, 2017, 122: 512-525.

[26] Tang FL, Zhang M, Xu JF et al. Pollution characteristics and health risk assessment of organochlorine pesticides( OCPs) in the water of lake qiandao and its major input Rivers. Chinese Journal of Environmental Science, 2014, 35(5): 17351741. [唐访良, 张明, 徐建芬等. 千岛湖库区及其主要人库河流水中有机氯农药残留污染特征及健康风险评价. 环境科学, 2014, 35(5): 1735-1741.]

[27] Lu Y. Distribution and sources of persistant organic pollutants in aquatic environment of Lake Taihu[Dissertation]. Hohhot: Inner Mogolia University, 2011. [鲁玥. 太湖水体中几种持久性有机污染物的时空分布研究 [学位论文]. 呼和 浩特: 内蒙古大学, 2011.]

[28] Wu M. Distribution of organochlorine pesticides before Danjiangkou Reservoir in the middle line of South-to-North Water Transfer Project [Dissertation]. Wuhan: Huazhong Normal University, 2014. [ 吴敏. 南水北调中线丹江口水库调水前 有机氯农药的分布研究 [学位论文]. 武汉: 华中师范大学, 2014.]

[29] Qu MX. Preliminary study on the pollution of persistent organic pollutants in polycyclic aromatic hydrocarbons in Baihua Lake [Dissertation]. Guiyang: Guizhou Normal University, 2006. [曲明昕. 百花湖水体中持久性有机污染物一一多 环芳烃的污染初步研究 [学位论文]. 贵阳: 贵州师范大学, 2006.]

[30] Li HL, Li GG, Gao H et al. Analysis and distribution of polycyclic aromatic hydrocarbons in Nansi Lake. Chemical Analytical Metrology, 2006, 15(6): 32-34. [李红莉, 李国刚, 高虹等. 南四湖水中多环芳烃的分析及分布研究. 化学分 析计量, 2006, 15(6): 32-34.]

[31] Liang YL, Liang GW, Jiang PC et al. Study on residues of organochlorine pesticides in surface water of West Lake in Hangzhou. Journal of China Jiliang University, 2008, 19(2): 174-177. [梁一灵, 梁国伟, 姜普查等. 杭州西湖表层水体 有机氯农药残留特性研究. 中国计量学院学报, 2008, (2): 174-177.]

[32] Lv HB. Pollution characteristics and kinetics of organochlorine pesticides (OCPs) in Jinshui River waters and sediments [Dissertation]. Jinan: Shandong Jianzhu University, 2015. [吕海波. 有机氯农药( OCPs) 在锦绣川水体与沉积物中的 污染特征及动力学研究 [学位论文]. 济南: 山东建筑大学, 2015.]

[33] Wang Y, Liu GJ, Liu RQ. Distribution and risk assessment of organochlorine pesticides in surface waters and sediments of Chaohu Lake area and rivers entering lakes. Environmental Chemistry, 2019, 38 (3): 669-678. [王元, 刘桂建, 刘荣 琼. 巢湖湖区及人湖河流表层水体、沉积物中有机氯农药分布及风险评价. 环境化学, 2019, 38(3): 669-678.]

[34] Chang S, Zhao XR, Fu Q et al. Distribution characteristics and risks of dissolved polycyclic aromatic hydrocarbons in waters of Yuqiao Reservoir Basin during water transfer. Environmental Science, 2016, 37(7): 2530-2538. [昌盛, 赵兴茹, 付青等. 输水期间于桥水库流域水体中溶解态多环芳烃的分布特征与风险. 环境科学, 2016, 37(7) : 2530-2538.]

[35] Christensen ER, Bzdusek PA. PAHs in sediments of the Black River and the Ashtabula River, Ohio: source apportionment by factor analysis. Water Research, 2005, 39 (4) : 511-524.

[36] Simpson CD, Mosi AA, Cullen WR et al. Composition and distribution of polycyclic aromatic hydrocarbon contamination in surficial marine sediments from Kitimat Harbor, Canada. Science of the Total Environment, 1996, 181(3) : 265-278.

[37] Liu CC, He JN, Qiu YL et al. Pollution characteristics of organochlorine pesticides in the Huangpu River water phase. Environmental Chemistry, 2017, 36(4):849-857. [刘翠翠, 何洁妮, 仇雁翎等. 黄浦江水相中有机氯农药的污染特征 分析. 环境化学, 2017, 36(4):849-857.]

[38 ] Liu CC, He JN, Qiu YL et al. Pollution characteristics of organochlorine pesticides in the Huangpu River water phase. Environmental Chemistry, 2017, 36(4):849-857. [刘翠翠, 何洁妮, 仇雁翎等. 黄浦江水相中有机氯农药的污染特征 分析. 环境化学, 2017, 36(4):849-857.]

[39] Kirsten H. United nations economic commission for Europe: Draft protocol to the convention on long-range transboundary air pollution on persistent organic pollutants. International Legal Materials, 1998, 37 (3) : 505-529. 
[40] Xi ZQ, Chu SG, Xu XB et al. Study on polychlorinated biphenyls in Donghu Lake. Oceanologia et Limnologia Sinica, 1998, 29(4) : 436-440. [习志群, 储少岗, 徐晓白等. 东湖水体中多氯联苯的研究. 海洋与湖沼, 1998, 29 (4) : 436-440.]

[41] Jiao L. Quantitative structure-activity relationship of PCB n-octanol-water partition coefficient. Journal of Northweste University: Natural Science Edition, 2011, 41(4): 623-627. [焦龙. 多氯联苯正辛醇-水分配系数的定量构效关系. 西北大 学学报: 自然科学版, 2011, 41(4): 623-627.]

[42] Zhong YS, Chen S, Cao Y et al. Determination of phthalates in water bodies of Beijing park and their distribution characteristics. China Environmental Monitoring, 2010, 26(3) : 60-64. [钟嶷盛, 陈莎, 曹莹等. 北京公园水体中邻苯二甲 酸酯类物质的测定及其分布特征. 中国环境监测, 2010, 26(3) : 60-64.]

[43] Qin J, Lu GH, Zhu ZL et al. Environmental and human distribution and ecotoxicological effects of polybrominated diphenyl ethers. Journal of Environment and Health, 2009, 26(8) : 740-743. [秦健, 陆光华, 朱正丽等. 多溴联苯醚的环境和 人体分布及生态毒理学效应. 环境与健康杂志, 2009, 26(8): 740-743.]

[44] Wang GS, Zhong ZQ, Wang FT. The hazard and pathway of PAH (polycyclic aromatic hydrocarbons). Shandong Environment, 2001, (2) : 41. [王桂山, 仲兆庆, 王福涛. PAH (多环芳烃) 的危害及产生的途径. 山东环境, 2001, (2): 41.]

[45] Yang Y, Liu XT, Wang XC et al. Progress in China's implementation of the stockholm convention on prevention and control of persistent organic pollutants. Contemporan Ecological Agriculture, 2011, (Z2): 133-137. [杨运, 刘晓肜, 王喜春 等. 中国履行斯德哥尔摩公约防治持久性有机污染物进展. 当代生态农业, 2011，(Z2)：133-137.]

[46] Wang ZF, Zhao ZY. Promoting sustainable development of agriculture by integrated prevention and control of POPs. Environmental Protection, 2010, (23) : 22-23. [王志芳, 赵子鹰. 以 POPs 综合防治促进农业可持续发展. 环境保护, 2010, (23): 22-23.]

[47] Yun XY, Yang YY, Li QX et al. Present situation and distribution characteristics of persistent organic pollutants in freshwater in China. Environmental Chemistry, 2013, 32(11): 2072-2081. [员晓燕, 杨玉义, 李庆孝等. 中国淡水环境中 典型持久性有机污染物 (POPs) 的污染现状与分布特征. 环境化学, 2013, 32(11): 2072-2081.]

[48] Yang GS, Ma RH, Zhang L et al. Current situation of lakes in China and major problems and protection strategies. J Lake Sci, 2010, 22(6) : 799-810. DOI: 10.18307/2010.0601. [ 杨桂山, 马荣华, 张路等. 中国湖泊现状及面临的重大问 题与保护策略. 湖泊科学, 2010, 22(6): 799-810.]

[49] Chen BL, Xuan XD, Zhu LZ et al. Distributions of polycyclic aromatic hydrocarbons in surface waters, sediments and soils of Hangzhou City, China. Water Research, 2004, 38(16) : 3558-3568.

[50] Qiao M, Huang SB, Wang ZJ. Partitioning characteristics of PAHs between sediment and water in a shallow lake. Journal of Soils and Sediments, 2008, 8(2): 69-73.

[51] Yang LP, Zhu LY, Liu ZT. Occurrence and partition of perfluorinated compounds in water and sediment from Liao River and Taihu Lake, China. Chemosphere, 2011, 83(6) : 806-814. DOI: 10.1016/j.chemosphere.2011.02.075.

[52] Yu H, Chang Y, Ye XY et al. Environmental evolution of polycyclic aromatic hydrocarbons in Baihua Lake waters of Guizhou province. Guizhou Science, 2010, 28(3): 85-89. [余洪, 常缨, 叶晓云等. 贵州百花湖水体中多环芳烃的环 境演化. 贵州科学, 2010, 28(3) : 85-89.]

[53] Yang L, Wang BX, Hou Y et al. Rapid determination of 16 polycyclic aromatic hydrocarbons in Dianchi Lake system by stir bar adsorption-thermal desorption-gas chromatography/mass spectrometry. Chinese Journal of Chromatography, 2007, 25(5) : 747-752. [ 杨蕾, 王保兴, 侯英等. 应用摚拌棒吸附萃取-热脱附-气相色谱/质谱法快速测定滇池水系中的 16 种多环芳烃. 色谱, 2007, 25(5): 747-752.]

[54] Wang YF, Ren SJ, Li MR et al. Residues and health risk assessment of polycyclic aromatic hydrocarbons in Guanting Reservoir. Journal of Beijing Normal University: Natural Science, 2015, 51(1) : 60-63. [王亚飞, 任姝娟, 李沫芯等. 官厅 水库水体多环芳烃残留特征及健康风险评价. 北京师范大学学报：自然科学版, 2015, 51(1) : 60-63.]

[55] Li JY, Shi WX, Cui Y et al. Pollution analysis and evaluation of heavy metals and polycyclic aromatic hydrocarbons in water and sediment of dishui Lake. Journal of Ecology and Rural Environment, 2016, 32(1) : 96-101. [李娟英, 石文瑄, 崔昱等. 滴水湖水体及沉积物中重金属和多环芳烃的污染分析与评价. 生态与农村环境学报, 2016, 32 (1): 96-101.]

[56] Liu H, Gan ZY, Zou S et al. Pollution characteristics and evaluation of organic pollutants in Yunlong Lake Waters. Envi- 
ronmental Science, 2013, 26(1) : 36-38, 47. [刘浩, 甘志永, 邹塞等. 云龙湖水体中有机类污染物污染特征与评 价. 环境科技, 2013, 26(1): 36-38, 47.]

[57] Li J, Zhang G, Qi SH. Characteristics and seasonal variations of bioavailable polycyclic aromatic hydrocarbons in Wuhu. Chongqing Environmental Science, 2003, (11): 108-109, 114. [李军, 张干, 祁士华. 麓湖中具生物有效性多环芳烃 的特征和季节变化. 重庆环境科学, 2003, (11) : 108-109, 114.]

[58] Tao YQ, Yu J, Liu XR et al. Factors affecting annual occurrence, bioaccumulation, and biomagnification of polycyclic aromatic hydrocarbons in plankton food webs of subtropical eutrophic lakes. Water Research, 2018, 132 : 1-11.

[59] Luo SX. Pollution status and source analysis of organic pollutants-polycyclic aromatic hydrocarbons in water and sediment of Hongfeng Lake [Dissertation]. Guiyang: Guizhou Normal University, 2005. [罗世霞. 红枫湖水体和沉积物中有机污 染物一多环芳烃的污染现状及源解析研究 [学位论文]. 贵阳: 贵州师范大学, 2005.]

[60] Zeng QF, Jeppesen E, Gu XH et al. Distribution, fate and risk assessment of PAHs in water and sediments from an aquaculture-and shipping-impacted subtropical lake, China. Chemosphere, 2018, 201 : 612-620.

[61] Wang XD, Zang SY, Zhang YH et al. Pollution characteristics and ecological risk assessment of polycyclic aromatic hydrocarbons in water and freshwater fish of Daqing Lake Group. Environmental Science, 2015, 36(11) : 4291-4301. [王晓迪, 藏淑英, 张玉红等. 大庆湖泊群水体和淡水鱼中多环芳烃污染特征及生态风险评估. 环境科学, 2015, 36(11): 4291-4301.]

[62] Shen BB, Wu JL, Zhao ZH. Organochlorine pesticides and polycyclic aromatic hydrocarbons in water and sediment of the Bosten Lake, Northwest China. Journal of Arid Land, 2017, 9(2) : 287-298.

[63] Liu Y, Shen JM., Chen ZL et al. Distribution of polycyclic aromatic hydrocarbons in surface water and sediment near a drinking water reservoir in Northeastern China. Environmental Science and Pollution Research International, 2013, 20(4) : $2535-2545$.

[64] Guo GH, Wu FC, He HP et al. Ecological risk assessment of organochlorine pesticides in surface waters of Lake Taihu, China. Human and Ecological Risk Assessment, 2013, 19(4) : 840-856.

[65] Qi XK. Study on the level, historical trend and migration law of organochlorine pesticides in Taihu Lake [Dissertation]. Beijing: Minzu University of China, 2012. [元学奎. 太湖有机氯农药的水平、历史趋势及迁移规律的研究 [学位论 文]. 北京: 中央民族大学, 2012.]

[66] He W, Qin N, He QS et al. Residues and potential risks of hexachlorocyclohexanes, HCHs, in surface water from Lake Chaohu during the high-water-level period. Acta Scientiae Circumstantiae, 2011, 31(5): 919-926. [何伟, 秦宁, 何玘霜 等. 丰水期巢湖表层水体六六六类农药的残留与风险. 环境科学学报, 2011, 31(5): 919-926.]

[67] Zhang M. Distribution characteristics and evaluation of typical persistent organic pollutants-organochlorine pesticides in waters of Chaohu Lake Basin [Dissertation]. Hefei: Anhui Agricultural University, 2009. [张明. 巢湖流域水体中典型持 久性有机污染物一有机氯农药的分布特征及评价 [学位论文]. 合肥: 安徽农业大学, 2009.]

[68] Yuan LX, Qi SH, Wu XG et al. Spatial and temporal variations of organochlorine pesticides (OCPs) in water and sediments from Honghu Lake, China. Journal of Geochemical Exploration, 2013, 132: 181-187.

[69] Su QK, Qi SH, Wu CX et al. Accumulation model of organochlorine pesticides in lake water and sediments by Honghu specialty aquatic products. Geological Science and Technology Information, 2007, 26(4)：85-90. [苏秋克, 祁士华, 吴 辰熙等. 洪湖特色水产品对湖水及沉积物中有机氯农药的积累模式. 地质科技情报, 2007, 26(4):85-90.]

[70] Ge DM, Han BP, Zheng W. Distribution and risk assessment of organochlorine pesticides in Weishan Lake waters. Anhui Agricultural Sciences, 2010, 38(22): 11987-11989, 12024. [葛冬梅, 韩宝平, 郑䂀. 微山湖水体中有机氯农药的分 布及风险评价. 安徽农业科学, 2010, 38(22): 11987-11989, 12024.]

[71] Shen YB, Zhang Y, Li CX et al. Residues and health risk assessment of organochlorine pesticides in Baihua Lake, Guizhou Province. Rural Eco-Environment, 2013, 29(3): 311-315. [沈烨冰, 张勇, 李存雄等. 贵州百花湖水体中有 机氯农药的残留及健康风险评价. 生态与农村环境学报, 2013, 29(3):311-315.]

[72] Yang J, Zhang WJ, Shen YF et al. Monitoring of organochlorine pesticides using PFU systems in Yunnan lakes and rivers, China. Chemosphere, 2007, 66(2): 219-225.

[73] Wan YW, Kang TF, Zhou ZL et al. Distribution characteristics and health risk assessment of organochlorine pesticides in Beijing Guanting Reservoir. Journal of Agro-Environment Science, 2009, 28(4): 803-807. [万译文, 康天放, 周忠亮 等. 北京官厅水库有机氯农药分布特征及健康风险评价. 农业环境科学学报, 2009, 28(4) : 803-807.] 
[74] Kang YH, Liu PB, Wang ZJ et al. Persistent organochlorine pesticide contamination in the water system of Guanting Reservoir-Yongding River, Beijing. J Lake Sci, 2003, 15(2) : 125-132. DOI: 10.18307/2003.0205. [康跃惠, 刘培斌, 王 子健等. 北京官厅水库-永定河水系水体中持久性有机氯农药污染. 湖泊科学, 2003, 15(2): 125-132.]

[75] Zhang WL, Zhang G, Qi SH et al. Preliminary study on organochlorine pesticides in the water and sediment of the Lake of Erhu and Yangzhuo Lake in Tibet. Geochemistry, 2003, 32(4): 363-367. [张伟玲, 张干, 祁士华等. 西藏错鄂湖和羊 卓雍湖水体及沉积物中有机氯农药的初步研究. 地球化学, 2003, 32(4) : 363-367.]

[76] Yao WF. Distribution characteristics and source analysis of persistent organic pollutants in sediments and waters of Gonghu Lake [Dissertation]. Changchun: Jilin Agricultural University, 2011. [姚威风. 持久性有机污染物在贡湖沉积物和水 体中的分布特征及源解析 [学位论文]. 长春: 吉林农业大学, 2011.]

[77] Wei ZQ. Biogeochemistry of organochlorine pollutants in Hongfeng Lake [Dissertation]. Guangzhou : Institute of Geochemistry, Chinese Academy of Sciences, 2006. [魏中青. 红枫湖有机氯污染物的生物地球化学 [学位论文]. 广州: 中国 科学院地球化学研究所, 2006.]

[78] Yang HD, Wang XY, Deng XF et al. Temporal and spatial distribution characteristics of organochlorine pesticides in surface waters of West Lake, Hangzhou. Journal of Zhejiang Agricultural Sciences, 2013, 25(1): 124-129. [杨洪达, 王祥 云, 邓勋飞等. 杭州西湖表层水体中有机氯农药的时空分布特征. 浙江农业学报, 2013, 25(1) : 124-129.]

[79] Wang XJ, Nie J, Yu GW et al. Microwave-assisted-demulsification-dispersive liquid-liquid microextraction coupled with gas chromatography-mass spectrometry for the determination of PAHs in water. Analytical Methods, 2018, 10 (42): 5105-5111.

[80] Zhang LL, Cui JS, Song T et al. Application of an AQUATOX model for direct toxic effects and indirect ecological effects assessment of polycyclic aromatic hydrocarbons (PAHs) in a plateau eutrophication lake, China. Ecological Modelling, 2018, 388: 31-44.

[81] Liang M. Investigation and analysis of water quality of main centralized drinking water sources in Jiangsu Province and research on protection countermeasures[Dissertation]. Nanjing: Nanjing Agricultural University, 2011. [梁蒙. 江苏省主要 集中式饮用水源地水质调查分析及保护对策的研究 [学位论文]. 南京: 南京农业大学, 2011.]

[82] Li SJ, Chen YN, Zhang JQ et al. The relationship of chromophoric dissolved organic matter parallel factor analysis fluorescence and polycyclic aromatic hydrocarbons in natural surface waters. Environmental Science and Pollution Research, 2018, 25 (2) : 1428-1438.

[83] Wu JJ. Distribution characteristics and source analysis of polycyclic aromatic hydrocarbons in the northern part of Taihu Lake [Dissertation]. Qingdao: Shandong University of Science and Technology, 2011. [武建军. 太湖北部多环芳烃的 分布特征及来源解析 [ 学位论文]. 青岛: 山东科技大学, 2011.]

[84] Sun QQ, Wu K. Temporal and spatial distribution and health risk of organochlorine pesticides in surface water of Chaohu Lake. Journal of Anqing Normal University: Natural Sciences, 2017, 23 (3) : 76-79. [孙倩倩, 吴克. 巢湖表层水中有 机氯农药的时空分布及健康风险. 安庆师范大学学报: 自然科学版, 2017, 23(3) : 76-79.]

[85] Sun QQ, Da CN, Wu K. Residue characteristics and source analysis of organochlorine pesticides in Chaohu Lake. Journal of Hefei University: Comprehensive Edition, 2017, 34(2): 69-73. [孙倩倩, 笪春年, 吴克. 巢湖水体中有机氯农药的 残留特征和来源分析. 合肥学院学报: 综合版, 2017, 34(2): 69-73.]

[86] Ren C. Distribution, source and ecological risk assessment of polycyclic aromatic hydrocarbons in Chaohu Lake Basin [Dissertation]. Hefei : Hefei University of Technology, 2015. [任琛. 巢湖流域多环芳烃的分布、来源与生态风险评价 [学位论文]. 合肥: 合肥工业大学, 2015.]

[87] Zou AH. Research on monitoring and pollution status of organic pollutants in the waters of the western half of Chaohu Lake [Dissertation]. Hefei: Hefei University of Technology, 2009. [邹爱红. 巢湖西半湖水体中有机污染物监测及污染现 状的研究 [学位论文]. 合肥: 合肥工业大学, 2009.]

[88] Li Q. Distribution characteristics and risks of PAHs in typical urban multi-media of Poyang Lake ecological economic zone [Dissertation]. Nanchang: Nanchang University, 2016. [李琴. 鄱阳湖生态经济区典型城市多介质中 PAHs 分布特征 及风险 [学位论文]. 南昌: 南昌大学, 2016.]

[89] Gong XY. Pollution characteristics and kinetics of organochlorine pesticides in lake waters and sediments [Dissertation]. Wuhan: China University of Geosciences, 2007. [ 龚香宜. 有机氯农药在湖泊水体和沉积物中的污染特征及动力学 研究[学位论文]. 武汉: 中国地质大学, 2007.] 
[90] Ge DM. Distribution, release and ecological risk of endogenous organochlorine pesticides in Weishan Lake [Dissertation]. Xuzhou : China University of Mining and Technology, 2014. [ 葛冬梅. 微山湖内源有机氯农药分布、释放及生态风险研 究[学位论文]. 徐州: 中国矿业大学, 2014.]

[91] Liu LL, Li J, Li MR et al. Residues and health risk assessment of organochlorine pesticides in Guanting Reservoir. Journal of Beijing Normal University: Natural Science, 2014, 50 (6): 657-661. [柳丽丽, 李剑, 李沫苑等. 官厅水库水体有 机氯农药残留特征及健康风险评价. 北京师范大学学报: 自然科学版, 2014, 50 (6) : 657-661.]

[92] Huang WY, Bao L, Teng MD et al. Distribution characteristics and environmental quality of organochlorine pesticides in water bodies of Wanfeng Lake. Environmental Science and Technology, 2016, 22(1): 52-57. [黄婉玉, 包亮, 滕明德等. 万峰湖水体中有机氯农药的分布特征与环境质量评价. 环保科技, 2016, 22(1)：52-57.]

[93] Wang YZ. Determination of 15 organochlorine pesticides in drinking water source in Yuqiao Reservoir water by solid phase extraction-gas chromatography-series tandem quadrupole mass spectrometry. Chinese Academy of Environmental Sciences, Nanjing Institute of Environmental Sciences, Ministry of Environmental Protection Ningxia Environmental Science Society. Proceedings of the 2nd National Watershed Ecological Protection and Water Pollution Control Symposium. China Environmental Science Society, Nanjing Institute of Environmental Sciences, Ministry of Environmental Protection, Ningxia Environmental Science Society: Chinese Society of Environmental Sciences, 2014: 2. [王乙震. 固相萃取-气相色谱三重串 联四级杆质谱法测定饮用水源地于桥水库水体中 15 种有机氯农药. 中国环境科学学会、环境保护部南京环境科 学研究所、宁夏环境科学学会. 第二届全国流域生态保护与水污染控制研讨会论文集. 中国环境科学学会、环境保 护部南京环境科学研究所、宁夏环境科学学会: 中国环境科学学会, 2014: 2.]

[94] Xu F, Qi SH. Pollution characteristics of organochlorine pesticides in waters of the Three Gorges Reservoir Area. Resources and Environment in the Yangtze Basin, 2012, 21(10): 1248-1253. [许峰, 祁士华. 三峡库区水体有机氯农药污染特 征. 长江流域资源与环境, 2012, 21(10): 1248-1253.]

[95] Lin L, Dong L, Li QY et al. Distribution and sources of polycyclic aromatic hydrocarbons and phthalates in water and sediment in the Three Gorges Reservoir Area. J Lake Sci, 2018, 30(3) : 660-667. DOI: 10.18307/2018.0308. [林莉, 董 否, 李青云等. 三峡库区水体和底泥中多环芳烃和邻苯二甲酸酯类分布和来源. 湖泊科学, 2018, 30(3): 660-667. ]

[96] Wang JX, Bi YH, Pfister G et al. Determination of PAH, PCB, and OCP in water from the Three Gorges Reservoir accumulated by semipermeable membrane devices (SPMD). Chemosphere, 2009, 75: 1119-1127.

[97] Wang JX, Henkelmann B, Bi YH et al. Temporal variation and spatial distribution of PAH in water of Three Gorges Reservoir during the complete impoundment period. Environmental Science and Pollution Research, 2013, 20 (10) : 7071-7079.

[98] Zhu YD, Yang YY, Liu MX et al. Concentration, distribution, source, and risk assessment of PAHs and heavy metals in surface water from the Three Gorges Reservoir, China. Human and Ecological Risk Assessment, 2015, 21(6) : 1593-1607.

[99] Wang YH, Qi SH, Wang W. Distribution characteristics of organochlorine pesticides in water and surface sediments of Wuhu Lake. Environmental Pollution and Control, 2007, 29(6): 415-418. [王英辉, 祁士华, 王伟. 汀汊湖水体和表层 沉积物中有机氯农药分布特征. 环境污染与防治, 2007, 29(6) : 415-418.]

[100] Dou W, Zhao ZX. Study on DDT and BHC pollution in Baiyangdian water body, sediment and carp. Journal of Environmental Science, 1998, 18(3) : 86-90. [窦薇, 赵忠宪. 白洋淀水体、底泥及鲫鱼体内 DDT、BHC 污染状况研究. 环境 科学学报, 1998, 18(3): 86-90.] 
附录 1 本文献中所涉及的湖泊位置及水域面积

Table S1 The location and water area of the lakes and reservoirs covered in this study

\begin{tabular}{|c|c|c|c|c|}
\hline & 湖泊名称 & 所处省份或直辖市 & 经纬度 & 水域面积 $/ \mathrm{km}^{2}$ \\
\hline \multirow[t]{31}{*}{ 东部湖区 } & 云龙湖 & 江苏 & $34^{\circ} 13^{\prime} \sim 34^{\circ} 14^{\prime} \mathrm{N}, 117^{\circ} 7^{\prime} \sim 117^{\circ} 10^{\prime} \mathrm{E}$ & 5.8 \\
\hline & 玄武湖 & 江苏 & $32^{\circ} 04^{\prime} \mathrm{N}, 118^{\circ} 47^{\prime} \mathrm{E}$ & 3.8 \\
\hline & 金牛湖 & 江苏 & $32^{\circ} 27^{\prime} \sim 32^{\circ} 31^{\prime} \mathrm{N}, 118^{\circ} 57^{\prime} \sim 119^{\circ} 1^{\prime} \mathrm{E}$ & 16.7 \\
\hline & 百家湖 & 江苏 & $31^{\circ} 56^{\prime} \mathrm{N}, 118^{\circ} 49^{\prime} \mathrm{E}$ & 1.7 \\
\hline & 固城湖 & 江苏 & $31^{\circ} 14^{\prime} \sim 31^{\circ} 18^{\prime} \mathrm{N}, 118^{\circ} 53^{\prime} \sim 118^{\circ} 57^{\prime} \mathrm{E}$ & 31.2 \\
\hline & 贡湖 & 江苏 & $31^{\circ} 24^{\prime} \mathrm{N}, 120^{\circ} 19^{\prime} \mathrm{E}$ & - \\
\hline & 太湖 & 江苏、浙江 & $30^{\circ} 55^{\prime} \sim 31^{\circ} 32^{\prime} 58^{\prime \prime} \mathrm{N}, 119^{\circ} 53^{\prime} \sim 120^{\circ} 36^{\prime} 10^{\prime \prime} \mathrm{E}$ & 2537.2 \\
\hline & 西湖 & 浙江 & $30^{\circ} 13^{\prime} \sim 30^{\circ} 15^{\prime} \mathrm{N}, 120^{\circ} 8^{\prime} \sim 120^{\circ} 9^{\prime} \mathrm{E}$ & 6.4 \\
\hline & 千岛湖 & 浙江 & $29^{\circ} 11^{\prime} \sim 30^{\circ} 02^{\prime} \mathrm{N}, 118^{\circ} 34^{\prime} \sim 119^{\circ} 15^{\prime} \mathrm{E}$ & 580 \\
\hline & 巢湖 & 安徽 & $31^{\circ} 25^{\prime} \sim 31^{\circ} 43^{\prime} \mathrm{N}, 117^{\circ} 16^{\prime} \sim 117^{\circ} 05^{\prime} \mathrm{E}$ & 786.0 \\
\hline & 洞庭湖 & 湖南 & $27^{\circ} 39^{\prime} \sim 29^{\circ} 51^{\prime} \mathrm{N}, 111^{\circ} 19^{\prime} \sim 113^{\circ} 34^{\prime} \mathrm{E}$ & 2614.4 \\
\hline & 洪湖 & 湖北 & $29^{\circ} 39^{\prime} \sim 30^{\circ} 12^{\prime} \mathrm{N}, 113^{\circ} 07^{\prime} \sim 114^{\circ} 05^{\prime} \mathrm{E}$ & 340.1 \\
\hline & 东湖 & 湖北 & $30^{\circ} 30^{\prime} \sim 30^{\circ} 35^{\prime} \mathrm{N}, 114^{\circ} 21^{\prime} \sim 114^{\circ} 27^{\prime} \mathrm{E}$ & 34.4 \\
\hline & 大冶湖 & 湖北 & $30^{\circ} 3^{\prime} \sim 30^{\circ} 8^{\prime} \mathrm{N}, 114^{\circ} 58^{\prime} \sim 115^{\circ} 12^{\prime} \mathrm{E}$ & 73.7 \\
\hline & 月湖 & 湖北 & $30^{\circ} 33^{\prime} \mathrm{N}, 114^{\circ} 15^{\prime} \mathrm{E}$ & 0.60 \\
\hline & 丹江口水库 & 湖北 & $32^{\circ} 43^{\prime} \mathrm{N}, 111^{\circ} 33^{\prime} \mathrm{E}$ & 1022.8 \\
\hline & 汀汉湖 & 湖北 & $30^{\circ} 40^{\prime} \sim 30^{\circ} 42^{\prime} \mathrm{N}, 113^{\circ} 38^{\prime} \sim 113^{\circ} 43^{\prime} \mathrm{E}$ & 86.7 \\
\hline & 南四湖 & 山东 & $34^{\circ} 27^{\prime} \sim 35^{\circ} 20^{\prime} \mathrm{N}, 116^{\circ} 34^{\prime} \sim 117^{\circ} 21^{\prime} \mathrm{E}$ & 930.2 \\
\hline & 微山湖 & 山东 & $34^{\circ} 26^{\prime} \sim 35^{\circ} 20^{\prime} \mathrm{N}, 116^{\circ} 34^{\prime} \sim 117^{\circ} 21^{\prime} \mathrm{E}$ & 531.2 \\
\hline & 于桥水库 & 天津 & $40^{\circ} 1^{\prime} \mathrm{N}, 17^{\circ} 32^{\prime} \mathrm{E}$ & 86.8 \\
\hline & 官厅水库 & 北京 & $40^{\circ} 26^{\prime} \mathrm{N}, 115^{\circ} 50^{\prime} \mathrm{E}$ & 130 \\
\hline & 滴水湖 & 上海 & $30^{\circ} 53^{\prime}-30^{\circ} 54^{\prime} \mathrm{N}, 121^{\circ} 55^{\prime}-121^{\circ} 57^{\prime} \mathrm{E}$ & 5.6 \\
\hline & 麓湖 & 广东 & $23^{\circ} 8^{\prime} \sim 23^{\circ} 9^{\prime} \mathrm{N}, 113^{\circ} 16^{\prime} \sim 113^{\circ} 17^{\prime} \mathrm{E}$ & 2.1 \\
\hline & 鄱阳湖 & 江西 & $28^{\circ} 23^{\prime} \sim 29^{\circ} 43^{\prime} \mathrm{N}, 115^{\circ} 48^{\prime} \sim 116^{\circ} 44^{\prime} \mathrm{E}$ & 3207.0 \\
\hline & 三峡水库 & 四川 & $30^{\circ} 49^{\prime} \mathrm{N}, 111^{\circ} 0^{\prime} \mathrm{E}$ & - \\
\hline & 白洋淀 & 河北 & $38^{\circ} 43^{\prime} \sim 39^{\circ} 02^{\prime} \mathrm{N}, 115^{\circ} 38^{\prime} \sim 116^{\circ} 07^{\prime} \mathrm{E}$ & 366 \\
\hline & 锦绣川水库 & 济南 & $36^{\circ} 30^{\prime} \mathrm{N}, 117^{\circ} 10^{\prime} \mathrm{E}$ & 166 \\
\hline & 新立城水库 & 吉林 & $43^{\circ} 51^{\prime} \sim 43^{\circ} 57^{\prime} \mathrm{N}, 125^{\circ} 43^{\prime} \sim 125^{\circ} 50^{\prime} \mathrm{E}$ & 1970 \\
\hline & 石头口门水库 & 吉林 & $43^{\circ} 47^{\prime} \sim 43^{\circ} 57^{\prime} \mathrm{N}, 125^{\circ} 43^{\prime} \sim 125^{\circ} 51^{\prime} \mathrm{E}$ & 4944 \\
\hline & 磨盘山水库 & 黑龙江 & $44^{\circ} 43^{\prime} \mathrm{N}, 127^{\circ} 26^{\prime} \mathrm{E}$ & 1151 \\
\hline & 大庆湖泊群 & 黑龙江 & $46^{\circ} 35^{\prime} \mathrm{N}, 125^{\circ} 1^{\prime} \mathrm{E}$ & - \\
\hline \multirow[t]{4}{*}{ 云贵高原湖区 } & 百花湖 & 贵州 & $26^{\circ} 37^{\prime} \sim 26^{\circ} 41^{\prime} \mathrm{N}, 106^{\circ} 29^{\prime} \sim 106^{\circ} 33^{\prime} \mathrm{E}$ & 14.5 \\
\hline & 红枫湖 & 贵州 & $26^{\circ} 24^{\prime} \sim 26^{\circ} 34^{\prime} \mathrm{N}, 106^{\circ} 20^{\prime} \sim 106^{\circ} 27^{\prime} \mathrm{E}$ & 57.2 \\
\hline & 万峰湖 & 贵州 & $24^{\circ} 46^{\prime} \sim 24^{\circ} 56^{\prime} \mathrm{N}, 105^{\circ} 0^{\prime} \sim 105^{\circ} 6^{\prime} \mathrm{E}$ & 816 \\
\hline & 云南高原湖泊群 & 云南 & $23^{\circ} 40^{\prime} \sim 27^{\circ} 50^{\prime} \mathrm{N}, 99^{\circ} 38^{\prime} \sim 102^{\circ} 34^{\prime} \mathrm{E}$ & - \\
\hline \multirow[t]{3}{*}{ 青藏一新疆湖区 } & 博斯腾湖 & 新疆 & $41^{\circ} 49^{\prime} \sim 42^{\circ} 08^{\prime} \mathrm{N}, 86^{\circ} 41^{\prime} \sim 87^{\circ} 27^{\prime} \mathrm{E}$ & 1004.3 \\
\hline & 错鄂湖 & 西藏 & $31^{\circ} 24^{\prime} \sim 31^{\circ} 41^{\prime} \mathrm{N}, 88^{\circ} 32^{\prime} \sim 88^{\circ} 50^{\prime} \mathrm{E}$ & 333.8 \\
\hline & 羊卓雍湖 & 西藏 & $28^{\circ} 46^{\prime} \sim 29^{\circ} 11^{\prime} \mathrm{N}, 90^{\circ} 22^{\prime} \sim 91^{\circ} 4^{\prime} \mathrm{E}$ & 650.5 \\
\hline
\end{tabular}

